\title{
On the Fredholm Property of the Stokes Operator in a Layer-Like Domain
}

\author{
S. A. Nazarov and K. Pileckas
}

\begin{abstract}
The Stokes problem is studied in the domain $\Omega \subset \mathbb{R}^{3}$ coinciding with the layer $\Pi=\left\{x=(y, z): y=\left(y_{1}, y_{2}\right) \in \mathbb{R}^{2}, z \in(0,1)\right\}$ outside some ball. It is shown that the operator of such problem is of Fredholm type; this operator is defined on a certain weighted function space $\mathcal{D}_{\beta}^{l}(\Omega)$ with norm determined by a stepwise anisotropic distribution of weight factors (the direction of $z$ is distinguished). The smoothness exponent $l$ is allowed to be a positive integer, and the weight exponent $\beta$ is an arbitrary real number except for the integer set $\mathbb{Z}$ where the Fredholm property is lost. Dimensions of the kernel and cokernel of the operator are calculated in dependence of $\beta$. It turns out that, at any admissible $\beta$, the operator index does not vanish. Based on the generalized Green formula, asymptotic conditions at infinity are imposed to provide the problem with index zero.
\end{abstract}

Keywords: Stokes equations, layer-like domains, Fredholm property, weighted spaces

AMS subject classification: 35Q30, 76D07

\section{Introduction}

Let $\Omega \subset \mathbb{R}^{3}$ be a domain coinciding outside the ball $B_{R_{0}}=\left\{x \in \mathbb{R}^{3}:|x|<R_{0}\right\}$ with the infinite layer

$$
\Pi=\left\{x=(y, z): y=\left(y_{1}, y_{2}\right) \in \mathbb{R}^{2}, z \in(0,1)\right\}
$$

For simplicity we assume the boundary $\partial \Omega$ to be smooth. Without loss of generality we also fix $R_{0}=1$. The set $\partial \Omega \backslash B_{1}$ contains infinite parts of two planes

$$
\begin{aligned}
& S^{(0)}=\left\{x: y \in \mathbb{R}^{2}, z=0\right\} \\
& S^{(1)}=\left\{x: y \in \mathbb{R}^{2}, z=1\right\}
\end{aligned}
$$

which form the boundary $\partial \Pi$ of the layer $\Pi$. We consider the Stokes system

$$
\left.\begin{array}{r}
-\nu \Delta \mathbf{u}+\nabla p=\mathbf{f} \\
-\operatorname{div} \mathbf{u}=g
\end{array}\right\} \quad(\text { in } \Omega)
$$

S. A. Nazarov: Inst. Mech. Eng. Prob., V.O. Bol'shoy pr. 61, St. Petersburg 199178, Russia K. Pileckas: Inst. Math. \& Inf., Akademijos 4, 2600 Vilnius, Lithuania 
with the boundary conditions

$$
\mathbf{u}=\mathbf{h} \quad(\text { on } \partial \Omega)
$$

where

$\mathbf{u}=\left(u_{1}, u_{2} . u_{3}\right)$ is the velocity field

$p$ is the pressure in the fluid

$\mathbf{f}=\left(f_{1}, f_{2}, f_{3}\right)$ is an external force

$g$ is a given scalar-valued function in $\Omega$

$\mathbf{h}$ is a given vector-valued function on $\partial \Omega$

$\nu$ is the constant coefficient of viscosity

$\nabla=\left(\frac{\partial}{\partial x_{1}}, \frac{\partial}{\partial x_{2}}, \frac{\partial}{\partial x_{3}}\right), \Delta=\nabla \cdot \nabla, \operatorname{div} \mathbf{u}=\nabla \cdot \mathbf{u}$

"." means the scalar product in $\mathbb{R}^{3}$.

In the previous paper [15] we have studied the properties of solutions $(\mathbf{u}, p)$ to problem $(1.2)$ - (1.3) in a two-parametric scale of weighted function spaces $\mathcal{D}_{\beta}^{l}(\Omega)$ and $\mathcal{R}_{\beta}^{l}(\Omega ; \partial \Omega)$ such that the mapping

$$
\mathcal{D}_{\beta}^{l}(\Omega) \ni(\mathbf{u}, p) \longmapsto \mathcal{S}_{\beta}^{l}(\mathbf{u}, p)=(\mathbf{f}, g, \mathbf{h}) \in \mathcal{R}_{\beta}^{l}(\Omega ; \partial \Omega)
$$

where $\mathcal{S}_{\beta}^{l}$ is the operator of the Stokes probem (1.2) - (1.3), becomes continuous. In (1.4) $l$ is a regularity index and $\beta$ a weight index. The exact definitions of these spaces and their properties are presented in Section 2. In terms of these spaces we have proved (see [15]) regularity results and a coercive estimate for the solution $(\mathbf{u}, p) \in L_{\beta}^{2}(\Omega) \times L_{\beta}^{2}(\Omega)$ where the latter space consists of functions with finite norm

$$
\left\|(\mathbf{u}, p) ; L_{\beta}^{2}(\Omega) \times L_{\beta}^{2}(\Omega)\right\|=\left(\int_{\Omega}\left(1+|y|^{2}\right)^{\beta}\left(|\mathbf{u}|^{2}+|p|^{2}\right) d x\right)^{\frac{1}{2}}
$$

Moreover, in [15] the asymptotic representation of the solution $(\mathbf{u}, p) \in L_{\beta}^{2}(\Omega) \times L_{\beta}^{2}(\Omega)$ is constructed.

In this paper we prove the Fredholm property of mapping (1.4), calculate the dimensions of the kernel and cokernel and therefore the index of the operator $\mathcal{S}_{\beta}^{l}$ in (1.4). Moreover, we derive integral formulae for the coefficients in the asymptotic representation of the solution, which lead to a generalized Green formula. This formula, in particular, furnishes asymptotic conditions at infinity (in the same way as in the paper [16] where the Stokes operator was studied in domains with cylindrical outlets to infinity). Note also that the Fredholm property of the Neumann problem operator for a second order elliptic equation in a layer-like domain was proved in [13].

Acknowledgement. This work was concluded during the authors' stay in Portugal in April - June, 1999, funded by grants from CMA/IST (Lisbon) and from CIM/Fundação Calouste Gulbenkian (Coimbra) - Thematic Term on Theoretical and Computational Fluid Dynamics. The Portuguese financial support is deeply acknowledged. 


\section{Weighted function spaces and preliminary results}

2.1 Function spaces. Let $G$ be an arbitrary domain in $\mathbb{R}^{n}(n \geq 2)$. As usual, denote by $C^{\infty}(G)$ the set of all indefinitely differentiable functions in $\bar{G}$ and let $C_{0}^{\infty}(G)$ be a subset of functions from $C^{\infty}(G)$ with compact supports in $G$. Further, $W^{l, 2}(G)(l \geq 0)$ indicates the Sobolev space and $W^{l-\frac{1}{2}, 2}(\partial G)(l \geq 1)$ the space of traces on the boundary $\partial G$ of functions from $W^{l, 2}(G)$. Besides, $W^{0,2}(G)=L^{2}(G)$ and $W_{l o c}^{l, 2}(G)$ consists of functions which belong to $W^{l, 2}(K)$ for every compact $K \subset \bar{G}$. The spaces of scalarand vector-valued functions are not distinguished in notations. The norm of an element $u$ in the function space $X$ is denoted by $\|u ; X\|$.

Let $\Omega \subset \mathbb{R}^{3}$ be a layer-like domain. Denote by $C_{0}^{\infty}(\bar{\Omega})$ the subset of functions from $C^{\infty}(\Omega)$ with compact supports in $\bar{\Omega}$ (functions from $C_{0}^{\infty}(\bar{\Omega})$ are equal to zero for large $|x|$, but not necessarily on $\partial \Omega$ ). We define the norm

$$
\left\|u ; V_{\beta}^{l}(\Omega)\right\|=\left(\int_{\Omega} \sum_{|\mu|=0}^{l}\left(1+r^{2}\right)^{\beta-l+|\mu|}\left|\nabla_{x}^{\mu} u(x)\right|^{2} d x\right)^{\frac{1}{2}}
$$

with homogeneous isotropic weight distribution. In (2.1) $r=|y| \quad\left(y \in \mathbb{R}^{2}\right), x=(y, z) \in$ $\mathbb{R}^{3}, \mu=\left(\mu_{1}, \mu_{2}, \mu_{3}\right)$ with $\mu_{1}, \mu_{2}, \mu_{3} \geq 0$ is a multi-index, and

$$
\nabla_{x}^{\mu} u=\frac{\partial^{|\mu|} u}{\partial x_{1}^{\mu_{1}} \partial x_{2}^{\mu_{2}} \partial x_{3}^{\mu_{3}}} \quad\left(|\mu|=\mu_{1}+\mu_{2}+\mu_{3}\right) .
$$

Analogously,

$$
\left\|u ; V_{\beta}^{l}\left(\mathbb{R}^{2}\right)\right\|=\left(\int_{\mathbb{R}^{2}} \sum_{|\gamma|=0}^{l}\left(1+r^{2}\right)^{\beta-l+|\gamma|}\left|\nabla_{y}^{\gamma} u(y)\right|^{2} d y\right)^{\frac{1}{2}}
$$

for functions $u$ depending on $y \in \mathbb{R}^{2}$ only where $\gamma=\left(\gamma_{1}, \gamma_{2}\right)$ with $\gamma_{1}, \gamma_{2} \geq 0$. The spaces $V_{\beta}^{l}(\Omega)$ and $V_{\beta}^{l}\left(\mathbb{R}^{2}\right)$ are the closures of $C_{0}^{\infty}(\bar{\Omega})$ and $C_{0}^{\infty}\left(\mathbb{R}^{2}\right)$ in norms $(2.1)$ and $(2.2)$, respectively. The spaces $V_{\beta}^{l}(G)$ with norm (2.1) or (2.2) were first employed by V. A. Kondratiev [1] (Kondratiev spaces) while treating solutions of elliptic boundary value problems in domains $G \subset \mathbb{R}^{n}(n \geq 2)$ with conical outlets to infinity (in this case the weight in $(2.1)$ should be changed to $\left.\left(1+|x|^{2}\right)\right)$.

Let $\beta \in \mathbb{R}$ and let $l, \kappa$ denote integers such that $l \geq 0$ and $0 \leq \kappa \leq l$. We introduce the space $\mathcal{V}_{\beta, \kappa}^{l}(\Omega)$ as the closure of $C_{0}^{\infty}(\bar{\Omega})$ in the norm

$$
\left\|v ; \mathcal{V}_{\beta, \kappa}^{l}(\Omega)\right\|=\left(\sum_{\alpha+|\gamma| \leq l} \int_{\Omega}\left(1+r^{2}\right)^{\beta+|\gamma|-(|\gamma|-\kappa)+}\left|\partial_{z}^{\alpha} \partial_{y}^{\gamma} v(y, z)\right|^{2} d y d z\right)^{\frac{1}{2}}
$$

where $\alpha \geq 0, \gamma=\left(\gamma_{1}, \gamma_{2}\right)$ with $\gamma_{1}, \gamma_{2} \geq 0,|\gamma|=\gamma_{1}+\gamma_{2}, \partial_{z}^{\alpha}=\frac{\partial^{\alpha}}{\partial z^{\alpha}}, \partial_{y}^{\gamma}=\frac{\partial^{|\gamma|}}{\partial y_{1}^{\gamma_{1}} \partial y_{2}^{\gamma_{2}}}$ and $(t)_{+}=\frac{t+|t|}{2}$ is the positive part of $t \in \mathbb{R}$. 
As it can be observed in (2.3), differentiation in $z$ does not change the weight multiplier. Differentiation in $y$ of order $|\gamma| \leq \kappa$ increases the weight exponent by $|\gamma|$ (i.e. reflects the Kondratiev distribution of weights [1]). At $|\gamma|=\kappa$ the weight distribution function has a step. Namely, the subtrahend $(|\gamma|-\kappa)_{+}$compensates the growth of the weight exponent provided $|\gamma|>\kappa$. In the case of a cone where all directions are equivalent such step-weighted spaces were introduced and investigated in $[4,5]$.

It is easy to see that

$$
V_{\beta}^{0}(\Omega)=\mathcal{V}_{\beta, 0}^{0}(\Omega)=L_{\beta}^{2}(\Omega)
$$

while

$$
\left\|v ; L_{\beta}^{2}(\Omega)\right\|=\left(\int_{\Omega}\left(1+r^{2}\right)^{\beta}|v(x)|^{2} d x\right)^{\frac{1}{2}}
$$

Finally, for $l \geq 1$ we introduce the trace space $\mathcal{V}_{\beta, \kappa}^{l-\frac{1}{2}}(\partial \Omega)$ of functions $v \in \mathcal{V}_{\beta, \kappa}^{l}(\Omega)$ supplied with the norm

$$
\left\|w ; \mathcal{V}_{\beta, \kappa}^{l-\frac{1}{2}}(\partial \Omega)\right\|=\inf \left\{\left\|v ; \mathcal{V}_{\beta, \kappa}^{l}(\Omega)\right\|: v=w \text { on } \partial \Omega\right\}
$$

The trace $w$ on $\partial \Omega$ of $v \in \mathcal{V}_{\beta, \kappa}^{l}(\Omega)$ is forgetting the normal direction $z$ and the weight distribution in the norm of $\mathcal{V}_{\beta, \kappa}^{l-\frac{1}{2}}(\partial \Omega)$ turns into an isotropic one while preserving the step property. This becomes evident after using an equivalent norm in $\mathcal{V}_{\beta, \kappa}^{l-\frac{1}{2}}(\partial \Omega)$.

Lemma 2.1 (see $[15])$. The norm $\left\|\zeta ; \mathcal{V}_{\beta, \kappa}^{l-\frac{1}{2}}(\partial \Omega)\right\|(\kappa \leq l)$ is equivalent to

$$
\begin{aligned}
\|\boldsymbol{\|}\| \|= & \left\{\left\|\zeta ; W^{l-\frac{1}{2}, 2}\left(\partial \Omega \cap B_{2}\right)\right\|^{2}\right. \\
& +\sum_{j=0}^{1}\left(\sum_{0 \leq|\gamma| \leq l-1} \int_{S^{(j)} \backslash B_{1}}\left(1+r^{2}\right)^{\beta+|\gamma|-(|\gamma|-\kappa)+}\left|\partial_{y}^{\gamma} \zeta(y)\right|^{2} d y\right. \\
& +\sum_{|\gamma|=l-1} \int_{S^{(j)} \backslash B_{1}} \int_{S^{(j)} \backslash B_{1}} \mid \partial_{y}^{\gamma}\left(\left(1+|y|^{2}\right)^{\beta+\kappa} \zeta(y)\right) \\
& \left.\left.-\left.\partial_{\tilde{y}}^{\gamma}\left(\left(1+|\tilde{y}|^{2}\right)^{\beta+\kappa} \zeta(\tilde{y})\right)\right|^{2}|y-\tilde{y}|^{-3} d y d \tilde{y}\right)\right\}^{\frac{1}{2}} .
\end{aligned}
$$

In (2.5) integration over $S_{0}$ and $S_{1}$ is performed separately in order to avoid confusion. The reason is that for large $r$ the boundary $\partial \Omega$ consists of two non-intersecting parts and the distance in $\mathbb{R}^{3}$ between two points $y$ and $\tilde{y}$ located one above the other on $S_{0}$ and $S_{1}$ is equal to 1 , while the distance between them on $\partial \Omega$ is $O(|y|)$. Interpretating the symbol $|y-\tilde{y}|$ appropriately one can delete the first sum over $j$ in (2.5) and replace $S_{j} \backslash B_{1}$ by $\partial \Omega \backslash B_{1}$.

2.2 Auxiliary propositions. Below we make use of basic properties of the spaces $\mathcal{V}_{\beta, \kappa}^{l}(\Omega)$ which we collect in this section. 
Lemma 2.2 (see [15]). Let $v \in \mathcal{V}_{\beta, \kappa}^{l}(\Omega) \quad(l \geq 1,0 \leq \kappa \leq l-1, \beta \in \mathbb{R})$. Then $\partial_{y} v \in \mathcal{V}_{\beta+1, \kappa-1}^{l-1}(\Omega)$ and $\partial_{z} v \in \mathcal{V}_{\beta, \kappa}^{l-1}(\Omega)$. There holds the inequality

$$
\left\|\partial_{y} v ; \mathcal{V}_{\beta+1, \kappa-1}^{l-1}(\Omega)\right\|+\left\|\partial_{z} v ; \mathcal{V}_{\beta, \kappa}^{l-1}(\Omega)\right\| \leq c\left\|v ; \mathcal{V}_{\beta, \kappa}^{l}(\Omega)\right\|
$$

\section{Lemma 2.3.}

(i) The embeddings

$$
\begin{aligned}
\mathcal{V}_{\beta, \kappa}^{l}(\Omega) \hookrightarrow \mathcal{V}_{\beta, \kappa}^{l-1}(\Omega) & (l \geq 1,0 \leq \kappa \leq l-1) \\
\mathcal{V}_{\beta_{1}, \kappa}^{l}(\Omega) \hookrightarrow \mathcal{V}_{\beta, \kappa}^{l}(\Omega) & \left(l \geq 0,0 \leq \kappa \leq l, \beta_{1}>\beta\right)
\end{aligned}
$$

are continuous.

(ii) If $l \geq 1,0 \leq \kappa \leq l-1$ and $\varepsilon>0$, then the embedding

$$
\mathcal{V}_{\beta, \kappa}^{l}(\Omega) \hookrightarrow \mathcal{V}_{\beta-\varepsilon, \kappa}^{l-1}(\Omega)
$$

is compact.

Proof. Continuity of the embeddings (2.6) - (2.7) follows from the definition of the norm (2.1). Moreover,

$$
\left\|u ; \mathcal{V}_{\beta-\varepsilon, \kappa}^{l-1}\left(\Omega \backslash B_{2 R}\right)\right\| \leq c R^{-\varepsilon}\left\|u ; \mathcal{V}_{\beta, \kappa}^{l}\left(\Omega \backslash B_{R}\right)\right\| .
$$

Since $\mathcal{V}_{\beta, \kappa}^{l}\left(\Omega \cap B_{2 R}\right)$ coincides with $W^{l, 2}\left(\Omega \cap B_{2 R}\right)$ algebraically and topologically, well known properties of Sobolev spaces show that the embedding operator (2.8) can be represented as sum of a small operator (as $R \rightarrow \infty$ ) and a compact one. Thus (2.8) is compact

Let us prove one simple interpolation result.

Lemma 2.4. Let $v \in\left[\mathcal{V}_{\beta, 0}^{1}(\Omega)\right]^{*}$, where $\left[\mathcal{V}_{\beta, 0}^{1}(\Omega)\right]^{*}$ is the dual space to $\mathcal{V}_{\beta, 0}^{1}(\Omega)$ with respect to the scalar product in $L^{2}(\Omega)$. Suppose that $\nabla v \in L_{-\beta}^{2}(\Omega)$. Then $v \in L_{-\beta}^{2}(\Omega)$ and

$$
\left\|v ; L_{-\beta}^{2}(\Omega)\right\|^{2} \leq c\left(\left\|v ;\left[\mathcal{V}_{\beta, 0}^{1}(\Omega)\right]^{*}\right\|^{2}+\left\|\nabla v ; L_{-\beta}^{2}(\Omega)\right\|^{2}\right) .
$$

Proof. Let us cover the domain $\Omega$ by the infinite union of "cubes"

$$
Q_{s, k}=\left\{x \in \Omega:\left|x_{1}-s\right|,\left|x_{2}-k\right| \leq \frac{1}{2}\right\} \quad(s, k \in \mathbb{Z}) .
$$

By $\left[17:\right.$ Chapter 3/Lemma 7.1], for any function $v \in W^{-1,2}\left(Q_{s, k}\right)$ with $\nabla v \in L^{2}\left(Q_{s, k}\right)$ there holds the inclusion $v \in L^{2}\left(Q_{s, k}\right)$ and the estimate

$$
\left\|v ; L^{2}\left(Q_{s, k}\right)\right\|^{2} \leq c\left(\left\|v ; W^{-1,2}\left(Q_{s, k}\right)\right\|^{2}+\left\|\nabla v ; L^{2}\left(Q_{s, k}\right)\right\|^{2}\right)
$$

with constant $c$ independent of $s, k \in \mathbb{Z}$. Let us multiply the last inequalities by ( $1+$ $\left.\left(s^{2}+k^{2}\right)\right)^{-\beta}$ and sum them over all $s, k \in \mathbb{Z}$. Taking into account that $\left(1+r^{2}\right)$ is equivalent to $\left(1+\left(s^{2}+k^{2}\right)\right)$ in $Q_{s, k}$, we obtain

$$
\left\|v ; L_{-\beta}^{2}(\Omega)\right\|^{2} \leq c\left(\sum_{k, s \in \mathbb{Z}}\left(1+\left(s^{2}+k^{2}\right)\right)^{-\beta}\left\|v ; W^{-1,2}\left(Q_{s, k}\right)\right\|^{2}+\left\|\nabla v ; L_{-\beta}^{2}(\Omega)\right\|^{2}\right) .
$$


Further, the equivalency of the norms $\left\|\eta\left(1+r^{2}\right)^{\beta / 2} ; W^{1,2}(\Omega)\right\|$ and $\left\|\eta ; \mathcal{V}_{\beta, 0}^{1}(\Omega)\right\|$ gives the inequality

$$
\sum_{k, s \in \mathbb{Z}}\left(1+\left(s^{2}+k^{2}\right)\right)^{-\beta}\left\|v ; W^{-1,2}\left(Q_{s, k}\right)\right\|^{2} \leq c\left\|v ;\left[\mathcal{V}_{\beta, 0}^{1}(\Omega)\right]^{*}\right\|^{2}
$$

which competes the proof of the lemma

2.3 Space $\mathcal{D}_{\beta}^{l}(\Omega)$ - the domain of the Stokes operator. We fix some weight and regularity indeces, i.e. numbers $\beta \in \mathbb{R}$ and $l \in \mathbb{N}_{0}$ and denote by $\mathcal{D}_{\beta}^{l}(\Omega)$ the space of vector functions $(\mathbf{u}, p)$ satisfying the inclusions

$$
\begin{aligned}
\mathbf{u}^{\prime} & \in \mathcal{V}_{\beta+1, l}^{l+1}(\Omega) & u_{3} & \in \mathcal{V}_{\beta+2, l-1}^{l+1}(\Omega) \\
p & \in \mathcal{V}_{\beta, l}^{l}(\Omega) & \partial_{z} p & \in \mathcal{V}_{\beta+2, l-1}^{l-1}(\Omega) .
\end{aligned}
$$

The norm in $\mathcal{D}_{\beta}^{l}(\Omega)$ is given by the formula

$$
\begin{aligned}
& \left\|(\mathbf{u}, p) ; \mathcal{D}_{\beta}^{l}(\Omega)\right\| \\
& \quad=\left\|\mathbf{u}^{\prime} ; \mathcal{V}_{\beta+1, l}^{l+1}(\Omega)\right\|+\left\|u_{3} ; \mathcal{V}_{\beta+2, l-1}^{l+1}(\Omega)\right\|+\left\|p ; \mathcal{V}_{\beta, l}^{l}(\Omega)\right\|+\left\|\partial_{z} p ; \mathcal{V}_{\beta+2, l-1}^{l-1}(\Omega)\right\|
\end{aligned}
$$

Such definition of the space $\mathcal{D}_{\beta}^{l}(\Omega)$ has been used in the paper [15]. For purporses of this paper it is more convenient to employ the following equivalent definition. Let us represent the pressure function $p$ as sum

$$
p(x)=p_{\perp}(y, z)+\bar{p}(y)
$$

where

$$
\bar{p}(y)=\int_{0}^{1} p(y, z) d z
$$

is the mean value of $p$ with respect to $z \in(0,1)$. The projection $p_{\perp}$ obviously has zero mean value:

$$
\bar{p}_{\perp}(y, z)=\bar{p}(y, z)-\overline{\bar{p}}(y)=\bar{p}(y)-\bar{p}(y)=0 .
$$

Moreover,

$$
\overline{\partial_{y} p_{\perp}(y, z)}=\overline{\partial_{y} p(y, z)}-\overline{\partial_{y} \bar{p}(y)}=\partial_{y} \bar{p}(y)-\partial_{y} \bar{p}(y)=0 .
$$

Hence by the one-dimensional Poincare inequality we obtain $p_{\perp} \in L_{\beta+2}^{2}(\Omega), \partial_{y} p_{\perp} \in$ $L_{\beta+3}^{2}(\Omega)$ and

$$
\begin{gathered}
\left\|p_{\perp} ; L_{\beta+2}^{2}(\Omega)\right\| \leq c\left\|\partial_{z} p_{\perp} ; L_{\beta+2}^{2}(\Omega)\right\|=c\left\|\partial_{z} p ; L_{\beta+2}^{2}(\Omega)\right\| \\
\left\|\partial_{y} p_{\perp} ; L_{\beta+3}^{2}(\Omega)\right\| \leq c\left\|\partial_{z} \partial_{y} p_{\perp} ; L_{\beta+3}^{2}(\Omega)\right\| .
\end{gathered}
$$

Thus $p_{\perp} \in \mathcal{V}_{\beta+2, l}^{l}(\Omega)$ and

$$
\left\|p_{\perp} ; \mathcal{V}_{\beta+2, l}^{l}(\Omega)\right\| \leq c\left\|\partial_{z} p ; \mathcal{V}_{\beta+2, l-1}^{l-1}(\Omega)\right\|
$$


For the mean value $\bar{p}$ we get the inclusion $\bar{p} \in V_{\beta+l}^{l}\left(\mathbb{R}^{2}\right)$ and the estimate

$$
\left\|\bar{p} ; V_{\beta+l}^{l}\left(\mathbb{R}^{2}\right)\right\| \leq c\left\|p ; \mathcal{V}_{\beta, l}^{l}(\Omega)\right\| .
$$

Therefore the space $\mathcal{D}_{\beta}^{l}(\Omega)$ may be redefined as space of all vector functions $(\mathbf{u}, p)$ such that $\mathbf{u}$ satisfies inclusions (2.9) and $p$ admits representation (2.12) with

$$
\left.\begin{array}{rl}
p_{\perp} & \in \mathcal{V}_{\beta+2, l}^{l}(\Omega) \\
\bar{p} & \in V_{\beta+l}^{l}\left(\mathbb{R}^{2}\right)
\end{array}\right\} .
$$

An equivalent norm in $\mathcal{D}_{\beta}^{l}(\Omega)$ is given by the formula

$$
\begin{aligned}
& \left\|(\mathbf{u}, p) ; \mathcal{D}_{\beta}^{l}(\Omega)\right\| \\
& \quad=\left\|\mathbf{u}^{\prime} ; \mathcal{V}_{\beta+1, l}^{l+1}(\Omega)\right\|+\left\|u_{3} ; \mathcal{V}_{\beta+2, l-1}^{l+1}(\Omega)\right\|+\left\|p_{\perp} ; \mathcal{V}_{\beta+2, l}^{l}(\Omega)\right\|+\left\|\bar{p} ; V_{\beta+l}^{l}\left(\mathbb{R}^{2}\right)\right\| .
\end{aligned}
$$

2.4 Space $\mathcal{R}_{\boldsymbol{\beta}}^{l}(\Omega ; \partial \Omega)$ - the range of the Stokes operator. The space $\mathcal{R}_{\beta}^{l}(\Omega ; \partial \Omega)$ $(l \geq 1)$ consists of triples $(\mathbf{f}, g, \mathbf{h})$ such that

$$
\left.\begin{array}{rl}
g & \in \mathcal{V}_{\beta+2, l-1}^{l}(\Omega) \\
\mathbf{h}^{\prime} & \in \mathcal{V}_{\beta+1, l}^{l+\frac{1}{2}}(\partial \Omega) \\
h_{3} & \in \mathcal{V}_{\beta+2, l-1}^{l+\frac{1}{2}}(\partial \Omega)
\end{array}\right\}
$$

while $\mathbf{f}$ admits the representation

$$
\mathbf{f}=\mathbf{f}_{0}+\partial_{z} \mathbf{f}_{1}+\nabla \psi
$$

with

$$
\left.\begin{array}{rl}
\mathbf{f}_{0} & \in \mathcal{V}_{\beta+2, l-1}^{l-1}(\Omega) \\
\mathbf{f}_{1}^{\prime} & \in \mathcal{V}_{\beta+1, l}^{l}(\Omega) \\
f_{13} & \in \mathcal{V}_{\beta+2, l-1}^{l}(\Omega) \\
\psi_{\perp} & \in \mathcal{V}_{\beta+2, l}^{l}(\Omega) \\
\bar{\psi} & \in V_{\beta+l}^{l}\left(\mathbb{R}^{2}\right)
\end{array}\right\} .
$$

The norm in $\mathcal{R}_{\beta}^{l}(\Omega ; \partial \Omega)$ is given by

$$
\begin{aligned}
& \left\|(\mathbf{f}, g, \mathbf{h}) ; \mathcal{R}_{\beta}^{l}(\Omega ; \partial \Omega)\right\| \\
& =\inf \left\{\left\|\mathbf{f}_{0} ; \mathcal{V}_{\beta+2, l-1}^{l-1}(\Omega)\right\|+\left\|\mathbf{f}_{1}^{\prime} ; \mathcal{V}_{\beta+1, l}^{l}(\Omega)\right\|\right. \\
& \left.\quad+\left\|f_{13} ; \mathcal{V}_{\beta+2, l-1}^{l}(\Omega)\right\|+\left\|\psi_{\perp} ; \mathcal{V}_{\beta+2, l}^{l}(\Omega)\right\|+\left\|\bar{\psi} ; V_{\beta+l}^{l}\left(\mathbb{R}^{2}\right)\right\|\right\} \\
& \quad+\left\|g ; \mathcal{V}_{\beta+2, l-1}^{l}(\Omega)\right\|+\left\|\mathbf{h}^{\prime} ; \mathcal{V}_{\beta+1, l}^{l+\frac{1}{2}}(\partial \Omega)\right\|+\left\|h_{3} ; \mathcal{V}_{\beta+2, l-1}^{l+\frac{1}{2}}(\partial \Omega)\right\|
\end{aligned}
$$

where the infimum is taken over all representations (2.16). From Lemmata 2.2 and 2.3 we derive the following assertions. 
Lemma 2.5. The embeddings

$$
\left.\begin{array}{rl}
\mathcal{R}_{\beta}^{l}(\Omega ; \partial \Omega) \hookrightarrow \mathcal{R}_{\beta}^{l-1}(\Omega ; \partial \Omega) \\
\mathcal{R}_{\beta_{1}}^{l}(\Omega ; \partial \Omega) \hookrightarrow \mathcal{R}_{\beta}^{l}(\Omega ; \partial \Omega)
\end{array}\right\} \quad\left(l \geq 1, \beta_{1}>\beta\right)
$$

are continuous.

Theorem 2.1. The operator $\mathcal{S}_{\beta}^{l}$ of problem (1.2) - (1.3),

$$
\mathcal{D}_{\beta}^{l}(\Omega) \ni(\mathbf{u}, p) \longmapsto \mathcal{S}_{\beta}^{l}(\mathbf{u}, p)=(\mathbf{f}, g, \mathbf{h}) \in \mathcal{R}_{\beta}^{l}(\Omega ; \partial \Omega)
$$

is continuous.

2.5 Coercive estimate for the solution of problem (1.2) - (1.3). The following result is proved in [15].

Theorem 2.2. Let $(\mathbf{u}, p) \in L_{\beta}^{2}(\Omega) \times L_{\beta}^{2}(\Omega)$ be the solution of problem $(1.2)-(1.3)$ with right-hand side $(\mathbf{f}, g) \in \mathcal{R}_{\beta}^{l}(\Omega ; \partial \Omega) \quad(l \geq 1, \beta \in \mathbb{R})$. Then $(\mathbf{u}, p) \in \mathcal{D}_{\beta}^{l}(\Omega)$ and

$$
\begin{aligned}
& \left\|(\mathbf{u}, p) ; \mathcal{D}_{\beta}^{l}(\Omega)\right\| \\
& \quad \leq c\left(\left\|(\mathbf{f}, g, \mathbf{h}) ; \mathcal{R}_{\beta}^{l}(\Omega ; \partial \Omega)\right\|+\left\|\mathbf{u} ; L_{\beta}^{2}(\Omega)\right\|+\left\|p_{\perp} ; L_{\beta}^{2}(\Omega)\right\|+\left\|\bar{p} ; L_{\beta}^{2}\left(\mathbb{R}^{2}\right)\right\|\right) .
\end{aligned}
$$

In order to prove the Fredholm property of mapping (2.19) we need to transform estimate (2.20) into

$$
\left\|(\mathbf{u}, p) ; \mathcal{D}_{\beta}^{l}(\Omega)\right\| \leq c\left(\left\|(\mathbf{f}, g, \mathbf{h}) ; \mathcal{R}_{\beta}^{l}(\Omega ; \partial \Omega)\right\|+\left\|K(\mathbf{u}, p) ; \mathcal{D}_{\beta}^{l}(\Omega)\right\|\right)
$$

where $K$ is a compact operator in $\mathcal{D}_{\beta}^{l}(\Omega)$. As shown in [15], the function $\bar{p} \in L_{\beta}^{2}\left(\mathbb{R}^{2}\right) \cap$ $W_{l o c}^{l, 2}\left(\mathbb{R}^{2}\right)$ satisfies the Poisson equation

$$
-\frac{1}{6} \Delta_{y}^{\prime} \bar{p}(y)=\mathcal{F}(y) \quad\left(y \in \mathbb{R}^{2}\right)
$$

where

$$
\begin{aligned}
\mathcal{F}(y) & =\mathcal{F}^{(1)}(y)+\operatorname{div}_{y}^{\prime} \mathcal{F}^{(2)}(y)+\Delta_{y}^{\prime} \mathcal{F}^{(3)}(y)+\Delta_{y}^{\prime} \mathcal{F}^{(0)}(y) \\
\mathcal{F}^{(0)}(y) & =\int_{0}^{1} \partial_{z} p(y, z)\left(\frac{1}{6} z-\frac{1}{2} z^{2}+\frac{1}{3} z^{3}\right) d z \\
\mathcal{F}^{(1)}(y) & =2 \nu \int_{0}^{1} g(y, z) d z \\
\mathcal{F}^{(2)}(y) & =-\int_{0}^{1} \mathbf{f}^{\prime}(y, z) z(z-1) d z \\
\mathcal{F}^{(3)}(y) & =-\nu \int_{0}^{1} \operatorname{div}_{y}^{\prime} \mathbf{u}^{\prime}(y, z) z(z-1) d z
\end{aligned}
$$


The inclusion $(\mathbf{f}, g, \mathbf{h}) \in \mathcal{R}_{\beta}^{l}(\Omega ; \partial \Omega)$ furnishes $\mathbf{f}^{\prime} \in L_{\beta+1}^{2}(\Omega), \operatorname{div}_{y}^{\prime} \mathbf{f}^{\prime} \in L_{\beta+2}^{2}(\Omega)$ and $g \in L_{\beta+2}^{2}(\Omega)$. Hence, $\mathcal{F}^{(1)} \in L_{\beta+2}^{2}\left(\mathbb{R}^{2}\right), \operatorname{div}_{y}^{\prime} \mathcal{F}^{(2)} \in L_{\beta+2}^{2}\left(\mathbb{R}^{2}\right)$ and

$$
\left\|\mathcal{F}^{(1)} ; L_{\beta+2}^{2}\left(\mathbb{R}^{2}\right)\right\|+\left\|\operatorname{div}_{y}^{\prime} \mathcal{F}^{(2)} ; L_{\beta+2}^{2}\left(\mathbb{R}^{2}\right)\right\| \leq c\left\|(\mathbf{f}, g, \mathbf{h}) ; \mathcal{R}_{\beta}^{l}(\Omega ; \partial \Omega)\right\|
$$

Further, $(\mathbf{u}, p) \in \mathcal{D}_{\beta}^{l}(\Omega)$ so that

$$
\begin{aligned}
\mathbf{u}^{\prime} & \in \mathcal{V}_{\beta+1, l}^{l+1}(\Omega) & \Delta_{y}^{\prime} \operatorname{div}_{y}^{\prime} \mathbf{u}^{\prime} & \in L_{\beta+3}^{2}(\Omega) \subset L_{\beta+2}^{2}(\Omega) \\
\partial_{z} p & \in L_{\beta+2}^{2}(\Omega) & \Delta_{y}^{\prime}\left(\partial_{z} p\right) & \in L_{\beta+4}^{2}(\Omega) \subset L_{\beta+2}^{2}(\Omega) .
\end{aligned}
$$

This implies $\Delta_{y}^{\prime} \mathcal{F}^{(0)} \in L_{\beta+2}^{2}\left(\mathbb{R}^{2}\right), \Delta_{y}^{\prime} \mathcal{F}^{(3)} \in L_{\beta+2}^{2}\left(\mathbb{R}^{2}\right)$ and

$$
\begin{aligned}
& \left\|\Delta_{y}^{\prime} \mathcal{F}^{(0)} ; L_{\beta+2}^{2}\left(\mathbb{R}^{2}\right)\right\|+\left\|\Delta_{y}^{\prime} \mathcal{F}^{(3)} ; L_{\beta+2}^{2}\left(\mathbb{R}^{2}\right)\right\| \\
& \quad \leq c\left(\left\|\Delta_{y}^{\prime} \operatorname{div}_{y}^{\prime} \mathbf{u}^{\prime} ; L_{\beta+2}^{2}(\Omega)\right\|+\left\|\Delta_{y}^{\prime}\left(\partial_{z} p\right) ; L_{\beta+2}^{2}(\Omega)\right\|\right)
\end{aligned}
$$

Thus,

$$
\mathcal{F}=\mathcal{F}^{(1)}+\operatorname{div}_{y}^{\prime} \mathcal{F}^{(2)}+\Delta_{y}^{\prime}\left(\mathcal{F}^{(0)}+\mathcal{F}^{(3)}\right) \in L_{\beta+2}^{2}\left(\mathbb{R}^{2}\right)
$$

and

$$
\begin{aligned}
& \left\|\mathcal{F} ; L_{\beta+2}^{2}\left(\mathbb{R}^{2}\right)\right\| \\
& \quad \leq c\left(\left\|(\mathbf{f}, g, \mathbf{h}) ; \mathcal{R}_{\beta}^{l}(\Omega)\right\|+\left\|\Delta_{y}^{\prime} \operatorname{div}_{y}^{\prime} \mathbf{u}^{\prime} ; L_{\beta+2}^{2}(\Omega)\right\|+\left\|\Delta_{y}^{\prime}\left(\partial_{z} p\right) ; L_{\beta+2}^{2}(\Omega)\right\|\right) .
\end{aligned}
$$

The punctured space $\mathbb{R}^{2} \backslash\{0\}$ might be interpreted as two-dimensional cone (a complete one) in $\mathbb{R}^{2}$ so that $\mathbb{R}^{2}$ is a domain with conical outlet to infinity. Therefore general theorems on elliptic problems in such domains can be applied while treating the solution $\bar{p}$ of equation $(2.22)$. It is known (see $[1,2,12]$ ) that such problems have the Fredholm property in the scale of Kondratie spaces $V_{\gamma}^{l}\left(\mathbb{R}^{2}\right)$ if and only if every power solution $w(y)=r^{-\lambda} \Psi(\varphi)$ of the corresponding homogeneous problem is trivial, provided that $\lambda$ lies on the line $\{\lambda \in \mathbb{C}: \operatorname{Re} \lambda=\gamma-l+1\} \quad\left((r, \varphi)\right.$ are polar coordinates in $\left.\mathbb{R}^{2}\right)$. For the Laplace operator (2.22) all power solutions consist of harmonic polynomials of orders $m \in \mathbb{N}_{0}$ and derivatives of the fundamental solution $\Gamma(y)=-\frac{1}{2 \pi} \ln |y|$. This information together with the general results (see $[1,2,12])$ and estimate (2.23) gives

Lemma 2.6. Let $\bar{p} \in L_{\beta}^{2}\left(\mathbb{R}^{2}\right) \cap W_{\text {loc }}^{l, 2}\left(\mathbb{R}^{2}\right) \quad\left(l \geq 2, \beta \notin \pm \mathbb{N}_{0}\right)$ be the solution of the Poisson equation (2.22). Then $\bar{p} \in V_{\beta+2}^{2}\left(\mathbb{R}^{2}\right)$ and there holds the inequality

$$
\begin{aligned}
\left\|\bar{p} V_{\beta+2}^{2}\left(\mathbb{R}^{2}\right)\right\| \leq & c\left(\left\|\mathcal{F} ; L_{\beta+2}^{2}\left(\mathbb{R}^{2}\right)\right\|+\left\|\mathcal{K}_{1} \bar{p} ; V_{\beta+2}^{2}\left(\mathbb{R}^{2}\right)\right\|\right) \\
\leq & c\left(\left\|(\mathbf{f}, g, \mathbf{h}) ; \mathcal{R}_{\beta}^{l}(\Omega ; \partial \Omega)\right\|+\left\|\Delta_{y}^{\prime} \operatorname{div}_{y}^{\prime} \mathbf{u}^{\prime} ; L_{\beta+2}^{2}(\Omega)\right\|\right. \\
& \left.+\left\|\Delta_{y}^{\prime}\left(\partial_{z} p\right) ; L_{\beta+2}^{2}(\Omega)\right\|+\left\|\mathcal{K}_{1} \bar{p} ; V_{\beta+2}^{2}\left(\mathbb{R}^{2}\right)\right\|\right)
\end{aligned}
$$

where $\mathcal{K}_{1}$ is a compact operator in $V_{\beta+2}^{2}\left(\mathbb{R}^{2}\right)$. 
Remark 2.1. Lemma 2.6 remains valid also for $l=1$ and $l=0$. However, because of the shortage of the regularity in these cases the Poisson equation (2.22) for $\bar{p}$ should be understood in the sence of distributions, i.e. the solution $\bar{p} \in L_{\beta}^{2}\left(\mathbb{R}^{2}\right)$ satisfies the integral identity

$$
\begin{aligned}
& -\frac{1}{6} \int_{\mathbb{R}^{2}} \bar{p}(y) \Delta_{y}^{\prime} \eta(y) d y \\
& \quad=\int_{\mathbb{R}^{2}}\left(\mathcal{F}^{(1)}(y) \eta(y)-\mathcal{F}^{(2)}(y) \cdot \nabla_{y}^{\prime} \eta(y)+\left(\mathcal{F}^{(0)}(y)+\mathcal{F}^{(3)}(y)\right) \Delta_{y}^{\prime} \eta(y)\right) d y
\end{aligned}
$$

for all $\eta \in C_{0}^{\infty}\left(\mathbb{R}^{2}\right)$ where

$$
\begin{aligned}
& \mathcal{F}^{(0)} \in L_{\beta+2}^{2}\left(\mathbb{R}^{2}\right) \subset L_{\beta+1}^{2}\left(\mathbb{R}^{2}\right) \\
& \mathcal{F}^{(1)} \in L_{\beta+2}^{2}\left(\mathbb{R}^{2}\right) \subset L_{\beta+1}^{2}\left(\mathbb{R}^{2}\right) \\
& \mathcal{F}^{(2)} \in L_{\beta+1}^{2}\left(\mathbb{R}^{2}\right) \\
& \mathcal{F}^{(3)} \in L_{\beta+2}^{2}\left(\mathbb{R}^{2}\right) \subset L_{\beta+1}^{2}\left(\mathbb{R}^{2}\right) .
\end{aligned}
$$

Since results analogous to Lemma 2.6 are true for the solution $\bar{p} \in L_{\beta}^{2}\left(\mathbb{R}^{2}\right)$ of the Poisson identity (2.25) (e.g. [2]: Section 6.3] and [12: Theorems 3.5.7 and 4.2.4]), we conclude the estimate

$$
\begin{aligned}
\left\|\bar{p} L_{\beta}^{2}\left(\mathbb{R}^{2}\right)\right\| \leq & c\left(\left\|(\mathbf{f}, g, \mathbf{h}) ; \mathcal{R}_{\beta}^{l}(\Omega ; \partial \Omega)\right\|+\left\|\operatorname{div}_{y}^{\prime} \mathbf{u}^{\prime} ; L_{\beta+1}^{2}(\Omega)\right\|\right. \\
& \left.+\left\|\partial_{z} p ; L_{\beta+1}^{2}(\Omega)\right\|+\left\|\tilde{\mathcal{K}}_{1} \bar{p} ; L_{\beta}^{2}\left(\mathbb{R}^{2}\right)\right\|\right)
\end{aligned}
$$

where $\tilde{\mathcal{K}}_{1}$ is a compact operator in $L_{\beta}^{2}\left(\mathbb{R}^{2}\right)$

First, let $l \geq 2$ and $\beta \notin \pm \mathbb{N}_{0}$. Using inequality (2.24) we can rewrite estimate (2.20) in the form

$$
\begin{aligned}
\left\|(\mathbf{u}, p) ; \mathcal{D}_{\beta}^{l}(\Omega)\right\| \leq & c\left(\left\|(\mathbf{f}, g, \mathbf{h}) ; \mathcal{R}_{\beta}^{l}(\Omega ; \partial \Omega)\right\|+\left\|\mathbf{u} ; L_{\beta}^{2}(\Omega)\right\|\right. \\
& +\left\|p_{\perp} ; L_{\beta}^{2}(\Omega)\right\|+\left\|\Delta_{y}^{\prime} \operatorname{div}_{y}^{\prime} \mathbf{u}^{\prime} ; L_{\beta+2}^{2}(\Omega)\right\| \\
& \left.+\left\|\Delta_{y}^{\prime}\left(\partial_{z} p\right) ; L_{\beta+2}^{2}(\Omega)\right\|+\left\|\mathcal{K}_{1} \bar{p} ; V_{\beta+2}^{2}\left(\mathbb{R}^{2}\right)\right\|\right) .
\end{aligned}
$$

By Lemma 2.2, $\Delta_{y}^{\prime} \operatorname{div}_{y}^{\prime} \mathbf{u}^{\prime} \in \mathcal{V}_{\beta+4, l-3}^{l-2}(\Omega)$ and $\Delta_{y}^{\prime}\left(\partial_{z} p\right) \in \mathcal{V}_{\beta+4, l-3}^{l-3}(\Omega)$. Moreover, by virtue of Lemma 2.3 the embeddings

$$
\begin{aligned}
\mathcal{V}_{\beta+4, l-3}^{l-2}(\Omega) & \hookrightarrow L_{\beta+2}^{2}(\Omega) \\
\mathcal{V}_{\beta+4, l-3}^{l-3}(\Omega) & \hookrightarrow L_{\beta+2}^{2}(\Omega) \\
\mathcal{V}_{\beta+1, l}^{l+1}(\Omega) & \hookrightarrow L_{\beta}^{2}(\Omega) \\
\mathcal{V}_{\beta+2, l-2}^{l+1}(\Omega) & \hookrightarrow L_{\beta}^{2}(\Omega) \\
\mathcal{V}_{\beta+2, l}^{l}(\Omega) & \hookrightarrow L_{\beta}^{2}(\Omega)
\end{aligned}
$$


are compact. Hence, there hold the inequalities

$$
\begin{aligned}
\left\|\Delta_{y}^{\prime} \operatorname{div}_{y}^{\prime} \mathbf{u}^{\prime} ; L_{\beta+2}^{2}(\Omega)\right\| & \leq c\left\|\mathcal{K}_{2} \mathbf{u}^{\prime} ; \mathcal{V}_{\beta+1, l}^{l+1}(\Omega)\right\| \\
\left\|\Delta_{y}^{\prime}\left(\partial_{z} p\right) ; L_{\beta+2}^{2}(\Omega)\right\| & \leq c\left\|\mathcal{K}_{3} p_{\perp} ; \mathcal{V}_{\beta+2, l}^{l}(\Omega)\right\| \\
\left\|\left(\mathbf{u}^{\prime}, u_{3}\right) ; L_{\beta}^{2}(\Omega) \times L_{\beta}^{2}(\Omega)\right\| & \leq c\left\|\mathcal{K}_{4}\left(\mathbf{u}^{\prime}, u_{3}\right) ; \mathcal{V}_{\beta+1, l}^{l+1}(\Omega) \times \mathcal{V}_{\beta+2, l-1}^{l+1}(\Omega)\right\| \\
\left\|p_{\perp} ; L_{\beta}^{2}(\Omega)\right\| & \leq c\left\|\mathcal{K}_{5} p_{\perp} ; \mathcal{V}_{\beta+2, l}^{l}(\Omega)\right\|
\end{aligned}
$$

where $\mathcal{K}_{i} \quad(i=2,3,4,5)$ are compact operators. Therefore from (2.27) estimate $(2.21)$ follows. In the cases $l=0$ and $l=1$ we analogously get estimate (2.21) using inequality (2.26) instead of (2.24). Thus, we have proved

Theorem 2.3. Let $(\mathbf{u}, p) \in \mathcal{D}_{\beta}^{l}(\Omega)$ be the solution of problem (1.2) - (1.3) with right-hand side $(\mathbf{f}, g, \mathbf{h}) \in \mathcal{R}_{\beta}^{l}(\Omega ; \partial \Omega) \quad\left(l \geq 1, \beta \in \mathbb{R} \backslash\left\{ \pm \mathbb{N}_{0}\right\}\right)$. Then estimate $(2.21)$ holds with $\mathcal{K}$ being a compact operator in $\mathcal{D}_{\beta}^{l}(\Omega)$.

2.6 Asymptotic representation of the solution. Let us formulate a result concerning the asymptotic behavior of the solution $(\mathbf{u}, p)$ of problem (1.2) - (1.3).

Theorem 2.4 (see [15]). Assume that

$$
(\mathbf{f}, g, \mathbf{h}) \in \mathcal{R}_{\beta+k}^{l}(\Omega ; \partial \Omega) \quad\left(l \geq 1, \beta \notin \pm \mathbb{N}_{0}, k \in \mathbb{N}\right) .
$$

Then the solution

$$
(\mathbf{u}, p) \in L_{\beta}^{2}(\Omega) \times L_{\beta}^{2}(\Omega)
$$

of problem $(1.2)-(1.3)$ admits the asymptotic representation

$$
\left(\begin{array}{c}
\mathbf{u} \\
p
\end{array}\right)=\chi(r) \sum_{-\beta-k-1<m<-\beta-1}\left(\begin{array}{c}
c_{m}^{+} \mathbf{u}_{m}^{+}(y, z)+c_{m}^{-} \mathbf{u}_{m}^{-}(y, z) \\
c_{m}^{+} p_{m}^{+}(y)+c_{m}^{-} p_{m}^{-}(y)
\end{array}\right)+\left(\begin{array}{c}
\tilde{\mathbf{u}} \\
\tilde{p}
\end{array}\right)
$$

where $\chi$ is a smooth cut-off function with $\chi(r)=1$ for $r \geq 2$ and $\chi(r)=0$ for $r \leq 1$,

$$
\begin{aligned}
& \mathbf{u}_{m}^{ \pm}(y, z)=\frac{1}{2 \nu} z(z-1) \nabla_{y}^{\prime} p_{m}^{ \pm}(y), \quad u_{3 m}^{ \pm}(y, z)=0, \quad p_{0}^{+}(y)=1, \quad p_{0}^{-}(y)=-\frac{1}{2 \pi} \ln r \\
& \left.\begin{array}{l}
p_{m}^{+}(y)=(2 \pi|m|)^{-\frac{1}{2}} r^{m} \cos (m \varphi) \\
p_{m}^{-}(y)=(2 \pi|m|)^{-\frac{1}{2}} r^{m} \sin (|m| \varphi)
\end{array}\right\}
\end{aligned}
$$

$c_{m}^{ \pm}\left(m \in \pm \mathbb{N}_{0}\right)$ are constants and $(\tilde{\mathbf{u}}, \tilde{p}) \in \mathcal{D}_{\beta+k}^{l}(\Omega)$. There holds the estimate

$$
\begin{aligned}
& \left\|(\tilde{\mathbf{u}}, \tilde{p}) ; \mathcal{D}_{\beta+k}^{l}(\Omega)\right\|+\sum_{-\beta-k-1<m<-\beta-1}\left(\left|c_{m}^{+}\right|+\left|c_{m}^{-}\right|\right) \\
& \quad \leq c\left(\left\|(\mathbf{f}, g, \mathbf{h}) ; \mathcal{R}_{\beta+k}^{l}(\Omega ; \partial \Omega)\right\|+\left\|\mathbf{u} ; L_{\beta}^{2}(\Omega)\right\|+\left\|p_{\perp} ; L_{\beta}^{2}(\Omega)\right\|+\left\|\bar{p} ; L_{\beta}^{2}\left(\mathbb{R}^{2}\right)\right\|\right) .
\end{aligned}
$$

Remark 2.2. Analogous asymptotic formulae were obtained also for second order scalar elliptic operators (see $[9,11]$ ) and for the Lame operator (see $[6-8,10]$ ). 
2.7 Green' formula. Let $(\mathbf{u}, p) \in \mathcal{D}_{\beta}^{l}(\Omega)$ and $(\mathbf{v}, q) \in C_{0}^{\infty}(\bar{\Omega})$. Then for the Stokes problem (1.2) - (1.3) there holds Green' formula

$$
\begin{aligned}
& \int_{\Omega}(-\nu \Delta \mathbf{u}+\nabla p) \cdot \mathbf{v} d x-\int_{\Omega} q \operatorname{div} \mathbf{u} d x+\int_{\partial \Omega} \mathbf{u} \cdot\left(\mathbf{n} q-\nu \partial_{n} \mathbf{v}\right) d s \\
& \quad=\int_{\Omega}(-\nu \Delta \mathbf{v}+\nabla q) \cdot \mathbf{u} d x-\int_{\Omega} p \operatorname{div} \mathbf{v} d x+\int_{\partial \Omega} \mathbf{v} \cdot\left(\mathbf{n} p-\nu \partial_{n} \mathbf{u}\right) d s .
\end{aligned}
$$

Here $\mathbf{n}$ is the unit vector of the outward normal to $\partial \Omega$ and $\partial_{n}=\frac{\partial}{\partial \mathbf{n}}$ denotes the derivative with respect to $\mathbf{n}$. Note that all integrals in $(2.33)$ are finite since $(\mathbf{v}, q)$ is identically zero for large $|x|$. It is not difficult to verify that the integrals in $(2.33)$ remain finite if $(\mathbf{v}, q) \in \mathcal{D}_{-\beta-2}^{l}(\Omega)$. Therefore by continuity we conclude the following assertion.

Lemma 2.7. Green' formula (2.33) holds true for any pairs $(\mathbf{u}, p) \in \mathcal{D}_{\beta}^{l}(\Omega)$ and $(\mathbf{v}, q) \in \mathcal{D}_{-\beta-2}^{l}(\Omega)$.

\section{The Fredholm property}

In this section we prove the main result of the paper: the Fredholm property of the Stokes operator $\mathcal{S}_{\beta}^{l}$, i.e. we prove that the range $\mathcal{S}_{\beta}^{l} \mathcal{D}_{\beta}^{l}(\Omega)$ is a closed subspace of $\mathcal{R}_{\beta}^{l}(\Omega ; \partial \Omega)$ and that

$$
\begin{array}{r}
\operatorname{dim} \operatorname{ker} \mathcal{S}_{\beta}^{l}<\infty \\
\operatorname{dim} \text { coker } \mathcal{S}_{\beta}^{l}<\infty .
\end{array}
$$

Theorem 3.1. The operator $\mathcal{S}_{\beta}^{l}(l \geq 1)$ of the Stokes problem (1.2) - (1.3) is of Fredholm type, if $\beta \notin \mathbb{Z}$. If $\beta \in \mathbb{Z}$, then the range of $\mathcal{S}_{\beta}^{l}$ is not closed.

Proof. The finite-dimensionality of ker $\mathcal{S}_{\beta}^{l}$ and the closedness of the range $\mathcal{S}_{\beta}^{l} \mathcal{D}_{\beta}^{l}(\Omega)$ follow from estimate (2.21) (see Theorem 2.3) and a lemma by J. Peetre (see [18] or [3: Lemma 2.5.1]).

Let us prove the finite-dimensionality of coker $\mathcal{S}_{\beta}^{l}$. We show that the subspace $\operatorname{ker}\left(\mathcal{S}_{\beta}^{l}\right)^{*}=\operatorname{coker} \mathcal{S}_{\beta}^{l}$ admits the representation

$$
\operatorname{coker} \mathcal{S}_{\beta}^{l}=\left\{\left(\mathbf{v}, q,\left.\left(\mathbf{n} q-\nu \partial_{n} \mathbf{v}\right)\right|_{\partial \Omega}\right):(\mathbf{v}, q) \in \operatorname{ker} \mathcal{S}_{-\beta-2}^{l}\right\} .
$$

Let us consider the bounded linear functional $F_{(\mathbf{v}, q)}$ given on $\mathcal{R}_{\beta}^{l}(\Omega ; \partial \Omega)$ by the formula

$$
\begin{gathered}
F_{(\mathbf{v}, q)}(\mathbf{f}, g, \mathbf{h})=\int_{\Omega} \mathbf{f} \cdot \mathbf{v} d x-\int_{\Omega} g q d x+\int_{\partial \Omega} \mathbf{h} \cdot\left(\mathbf{n} q-\nu \partial_{n} \mathbf{v}\right) d s \\
(\mathbf{v}, q) \in \mathcal{D}_{-\beta-2}^{l}(\Omega) .
\end{gathered}
$$

If $(\mathbf{f}, g, \mathbf{h}) \in \mathcal{S}_{\beta}^{l} \mathcal{D}_{\beta}^{l}(\Omega)$ and $(\mathbf{v}, q) \in \operatorname{ker} \mathcal{S}_{-\beta-2}^{l}$, then from Green's formula (2.33) it follows that $F_{(\mathbf{v}, q)}(\mathbf{f}, g, \mathbf{h})=0$. Thus $F_{(\mathbf{v}, q)}$ is orthogonal to $\mathcal{S}_{\beta}^{l} \mathcal{D}_{\beta}^{l}(\Omega)$ and therefore $F_{(\mathbf{v}, q)} \in \operatorname{ker}\left(\mathcal{S}_{\beta}^{l}\right)^{*}$. Hence we have proved the inclusion

$$
\left\{\left(\mathbf{v}, q,\left.\left(\mathbf{n} q-\nu \partial_{n} \mathbf{v}\right)\right|_{\partial \Omega}\right):(\mathbf{v}, q) \in \operatorname{ker} \mathcal{S}_{-\beta-2}^{l}\right\} \subset \operatorname{ker}\left(\mathcal{S}_{\beta}^{l}\right)^{*}
$$


In order to prove the inverse inclusion we first consider the case $l=1$ and introduce the operator $\mathcal{S}_{\beta}^{*}$ adjoint to $\mathcal{S}_{\beta}$ (with respect to the scalar product in $L^{2}(\Omega)^{4} \times L^{2}(\partial \Omega)^{3}$ ). For brevity we write $\mathcal{S}_{\beta}, \mathcal{D}_{\beta}(\Omega)$ etc., omitting the regularity index $l=1$. We mention as well known fact (see, e.g., $[3,19])$ that the operator $\mathcal{S}_{\beta}^{*}$ acts on the space of distributions by the formula

$$
\mathcal{R}_{\beta}(\Omega ; \partial \Omega)^{*} \ni(\mathbf{v}, q, \mathbf{w}) \longmapsto \mathcal{S}_{\beta}^{*}(\mathbf{v}, q, \mathbf{w})=S\left(\pi_{\Omega} \mathbf{v}, \pi_{\Omega} q\right)+\mathbf{w} \otimes \delta_{\partial \Omega}
$$

Here $\pi_{\Omega} \mathbf{v}$ and $\pi_{\Omega} q$ are the extensions of $\mathbf{v}$ and $q$, respectively, by zero from $\Omega$ to the entire $\mathbb{R}^{3}, \delta_{\partial \Omega}$ is the Dirac function concentrated on $\partial \Omega$ so that $\mathbf{w} \otimes \delta_{\partial \Omega}$ is the distribution defined by the formula

$$
\left(\mathbf{w} \otimes \delta_{\partial \Omega}, \varphi\right)_{\mathbb{R}^{3}}=(\mathbf{w}, \varphi)_{\partial \Omega} \quad\left(\varphi \in C_{0}^{\infty}\left(\mathbb{R}^{3}\right)\right)
$$

where $(\cdot, \cdot)_{\partial \Omega}$ denotes the scalar product in $L^{2}(\partial \Omega)$, and

$$
S\left(\pi_{\Omega} \mathbf{v}, \pi_{\Omega} q\right)=\left(-\nu \Delta \pi_{\Omega} \mathbf{v}+\nabla \pi_{\Omega} q ;-\operatorname{div} \pi_{\Omega} \mathbf{v}\right)
$$

is the Stokes operator (1.2). Note that due to Green's formula (2.33) this operator is formally self-adjoint.

Let $\omega, \widehat{\omega}$ be two neighbourhoods of a point in $\bar{\Omega}$ and $\bar{\omega} \subset \widehat{\omega}$. If the right-hand side $\mathbf{U}=\left(U_{1}, U_{2}, U_{3}, U_{4}\right)$ of the equation

$$
\mathcal{S}_{\beta}^{*}(\mathbf{v}, q, \mathbf{w})=\mathbf{U} \in \mathcal{D}_{\beta}(\Omega)^{*}
$$

belongs to $H^{s}(\Omega \cap \widehat{\omega})^{3} \times H^{s+1}(\Omega \cap \widehat{\omega})$, then first $(\mathbf{v}, q)$ belongs to $H^{s+2}(\Omega \cap \omega)^{3} \times$ $H^{s+1}(\Omega \cap \omega)$, second it satisfies the relations $S(\mathbf{v}, q)=\mathbf{U}$ in $\Omega \cap \omega$ and $\mathbf{v}=0$ on $\partial \Omega \cap \omega$, and third $\mathbf{w}$ coincides with the trace of $\left(\mathbf{n} q-\nu \partial_{n} \mathbf{v}\right)$ on $\partial \Omega \cap \omega$ (see [19] and [3: Chapter 2.5.3]). Since $\operatorname{ker} \mathcal{S}_{\beta}^{*}$ contains the solutions $(\mathbf{v}, q, \mathbf{w}) \in \mathcal{R}_{\beta}(\Omega ; \partial \Omega)^{*}$ of the homogeneous equation (3.4) (i.e. $\mathbf{U}=0)$, we conclude that $(\mathbf{v}, q) \in C_{l o c}^{\infty}(\bar{\Omega})$ solves the homogeneous Stokes problem (1.2) - (1.3) and $\mathbf{w}$ is the trace of $\left(\mathbf{n} q-\nu \partial_{n} \mathbf{v}\right)$ on $\partial \Omega$. Further, by definition $\mathcal{R}_{\beta}(\Omega ; \partial \Omega)$ contains the subspace

$$
\mathbf{R}=L_{\beta+2}^{2}(\Omega)^{3} \times \mathcal{V}_{\beta+2,0}^{1}(\Omega) \times \mathcal{V}_{\beta+1,1}^{\frac{3}{2}}(\partial \Omega)^{2} \times \mathcal{V}_{\beta+2,0}^{\frac{3}{2}}(\partial \Omega)
$$

(we assume that $\mathbf{f}_{1}=0$ and $\psi=0$ in representation $(2.16)$ for $\mathbf{f}$, i.e. $\mathbf{f}=\mathbf{f}_{0}$ ). Consequently, $\mathcal{R}_{\beta}(\Omega ; \partial \Omega)^{*} \subset \mathbf{R}^{*}$. The first two factors in $\mathbf{R}^{*}$ coinside with $L_{-\beta-2}^{2}(\Omega)^{3} \times$ $\left[\mathcal{V}_{\beta+2,0}^{1}(\Omega)\right]^{*}$ and hence we have $\mathbf{v} \in L_{-\beta-2}^{2}(\Omega)^{3}$ and $q \in\left[\mathcal{V}_{\beta+2,0}^{1}(\Omega)\right]^{*}$.

Let us show that $q$ belongs to $L_{-\beta-2}^{2}(\Omega)$. Denote by $\zeta_{\rho}$ the smooth cut-off function with $\zeta_{\rho}(r)=1$ for $r \leq \rho, \zeta_{\rho}(r)=0$ for $r \geq 2 \rho$ and

$$
\left.\begin{array}{rl}
\left|\nabla \zeta_{\rho}(r)\right| & \leq c\left(1+r^{2}\right)^{-\frac{1}{2}} \\
\left|\nabla \nabla \zeta_{\rho}(r)\right| & \leq c\left(1+r^{2}\right)^{-1}
\end{array}\right\}
$$


with constant $c$ independent of $\rho$ and $r$. We multiply the homogeneous Stokes equations (1.2) by $\zeta_{\rho}(r)^{2}\left(1+r^{2}\right)^{-\beta-1} \mathbf{v}(x)$ and integrate by parts in $\Omega$ :

$$
\begin{aligned}
\nu \int_{\Omega} \zeta_{\rho}(r)^{2}\left(1+r^{2}\right)^{-\beta-1}|\nabla \mathbf{v}(x)|^{2} d x \\
=\int_{\Omega} q \mathbf{v}(x) \cdot \nabla\left[\zeta_{\rho}(r)^{2}\left(1+r^{2}\right)^{-\beta-1}\right] d x \\
\quad-\nu \int_{\Omega} \nabla \mathbf{v}(x) \cdot \mathbf{v}(x) \nabla\left[\zeta_{\rho}(r)^{2}\left(1+r^{2}\right)^{-\beta-1}\right] d x \\
=I_{1}+I_{2}
\end{aligned}
$$

Using (3.5) it is easy to show that

$$
\left|I_{2}\right| \leq \frac{\nu}{4} \int_{\Omega} \zeta_{\rho}(r)^{2}\left(1+r^{2}\right)^{-\beta-1}|\nabla \mathbf{v}(x)|^{2} d x+c(\nu) \int_{\Omega}\left(1+r^{2}\right)^{-\beta-2}|\mathbf{v}(x)|^{2} d x .
$$

For the first summand $I_{1}$ we get

$$
\begin{aligned}
\left|I_{1}\right| \leq & \left\|q ;\left[\mathcal{V}_{\beta+2,0}^{1}(\Omega)\right]^{*}\right\|\left\|\mathbf{v} \nabla\left[\zeta_{\rho}(r)^{2}\left(1+r^{2}\right)^{-\beta-1}\right] ; \mathcal{V}_{\beta+2,0}^{1}(\Omega)\right\| \\
\leq & c\left\|q ;\left[\mathcal{V}_{\beta+2,0}^{1}(\Omega)\right]^{*}\right\| \\
& \times\left(\int_{\Omega}\left(1+r^{2}\right)^{-\beta-2}|\mathbf{v}|^{2} d x+\nu \int_{\Omega} \zeta_{\rho}^{2}\left(1+r^{2}\right)^{-\beta-1}|\nabla \mathbf{v}|^{2} d x\right)^{\frac{1}{2}} \\
\leq & \frac{\nu}{4} \int_{\Omega} \zeta_{\rho}^{2}\left(1+r^{2}\right)^{-\beta-1}|\nabla \mathbf{v}|^{2} d x \\
& +c(\nu)\left(\left\|q ;\left[\mathcal{V}_{\beta+2,0}^{1}(\Omega)\right]^{*}\right\|^{2}+\int_{\Omega}\left(1+r^{2}\right)^{-\beta-2}|\mathbf{v}|^{2} d x\right)
\end{aligned}
$$

Substituting (3.7), (3.8) into (3.6) we derive the estimate

$$
\begin{aligned}
\int_{\Omega} \zeta_{\rho}^{2}\left(1+r^{2}\right)^{-\beta-1}|\nabla \mathbf{v}|^{2} d x & \leq c\left(\left\|q ;\left[\mathcal{V}_{\beta+2,0}^{1}(\Omega)\right]^{*}\right\|^{2}+\int_{\Omega}\left(1+r^{2}\right)^{-\beta-2}|\mathbf{v}|^{2} d x\right) \\
& <\infty
\end{aligned}
$$

with constant $c$ independent of $\rho$. Passing in (3.9) $\rho \rightarrow \infty$, we get $\nabla \mathbf{v} \in L_{-\beta-1}^{2}(\Omega)$. Since the solution $(\mathbf{v}, p)$ is smooth, from local estimates it follows (see [15: Proof of Lemma 3.1]) that $\nabla q \in L_{-\beta-1}^{2}(\Omega) \subset L_{-\beta-2}^{2}(\Omega)$ and

$$
\left\|\nabla q ; L_{-\beta-1}^{2}(\Omega)\right\| \leq c\left\|\nabla \mathbf{v} ; L_{-\beta-1}^{2}(\Omega)\right\|
$$

By Lemma 2.4 we conclude that $q \in L_{-\beta-2}^{2}(\Omega)$ and $\left\|q ; L_{-\beta-2}^{2}(\Omega)\right\| \leq c\left(\left\|q ;\left[\mathcal{V}_{\beta+2,0}^{1}(\Omega)\right]^{*}\right\|+\left\|\nabla q ; L_{-\beta-2}^{2}(\Omega)\right\|\right)<\infty$ 
Thus the solution $(\mathbf{v}, p)$ of the homogeneous Stokes problem (1.2) - (1.3) belongs to $L_{-\beta-2}^{2}(\Omega)^{3} \times L_{-\beta-2}^{2}(\Omega)$. By Theorem $2.2,(\mathbf{v}, p)$ belongs to $\mathcal{D}_{-\beta-2}(\Omega)$ and hence

$$
\operatorname{ker} \mathcal{S}_{\beta}^{*} \subset\left\{\left(\mathbf{v}, q,\left.\left(\mathbf{n} q-\nu \partial_{n} \mathbf{v}\right)\right|_{\partial \Omega}\right):(\mathbf{v}, q) \in \operatorname{ker} \mathcal{S}_{-\beta-2}\right\} .
$$

Formulae (3.3) and (3.10) prove representation (3.1) of coker $\mathcal{S}_{\beta}$. Since the numbers $\beta$ and $-\beta-2$ belong to the prohibited set $\mathbb{Z}$ simultaneously, $\operatorname{dim} \operatorname{ker} \mathcal{S}_{-\beta-2}<\infty$ and the finite-dimensionality of coker $\mathcal{S}_{\beta}$ is proved. Moreover, from (3.2) and Green's formula (2.33) we derive the following compatibility conditions for the Stokes problem (1.2) (1.3):

$$
\int_{\Omega} \mathbf{f} \cdot \mathbf{v} d x-\int_{\Omega} g q d x+\int_{\partial \Omega} \mathbf{h} \cdot\left(\mathbf{n} q-\nu \partial_{n} \mathbf{v}\right) d s=0
$$

for all $(\mathbf{v}, p) \in \operatorname{ker} \mathcal{S}_{-\beta-2}$.

Let us consider the case $l>1$. Assume that $(\mathbf{f}, g, \mathbf{h}) \in \mathcal{R}_{\beta}^{l}(\Omega ; \partial \Omega) \subset \mathcal{R}_{\beta}^{1}(\Omega ; \partial \Omega)$ with $\beta \notin \mathbb{Z}$. If the right-hand side (f, $g, \mathbf{h})$ satisfies the compatibility conditions (3.11), then there exists a solution $(\mathbf{u}, p) \in \mathcal{D}_{\beta}^{1}(\Omega)$ of problem (1.2) - (1.3). By virtue of Theorem 2.2 we get $(\mathbf{u}, p) \in \mathcal{D}_{\beta}^{l}(\Omega)$. This means that $(\mathbf{f}, g, \mathbf{h})$ is orthogonal to $\operatorname{ker}\left[\mathcal{S}_{\beta}^{l}\right]^{*}$. By the Hahn-Banach theorem this gives

$$
\operatorname{ker}\left[\mathcal{S}_{\beta}^{l}\right]^{*} \subset\left\{\left(\mathbf{v}, q,\left.\left(\mathbf{n} q-\nu \partial_{n} \mathbf{v}\right)\right|_{\partial \Omega}\right):(\mathbf{v}, q) \in \operatorname{ker} \mathcal{S}_{-\beta-2}^{1}\right\} .
$$

Since by Theorem $2.2 \operatorname{ker} \mathcal{S}_{-\beta-2}^{1}=\operatorname{ker} \mathcal{S}_{-\beta-2}^{l}$, the last relation together with (3.3) furnishes

$$
\operatorname{ker}\left[\mathcal{S}_{\beta}^{l}\right]^{*}=\left\{\left(\mathbf{v}, q,\left.\left(\mathbf{n} q-\nu \partial_{n} \mathbf{v}\right)\right|_{\partial \Omega}\right):(\mathbf{v}, q) \in \operatorname{ker} \mathcal{S}_{-\beta-2}^{l}\right\} .
$$

Thus in the case $\beta \notin \mathbb{Z}$

$$
\operatorname{dim} \operatorname{ker}\left[\mathcal{S}_{\beta}^{l}\right]^{*}=\operatorname{dim} \operatorname{ker} \mathcal{S}_{-\beta-2}^{l}<\infty .
$$

This proves the Fredholm property for $\mathcal{S}_{\beta}^{l}$ with $l>1$ and $\beta \notin \mathbb{Z}$.

Consider now the case $\beta \in \mathbb{Z}$. Since $\mathcal{D}_{\beta}^{l}(\Omega) \subset \mathcal{D}_{\beta-\varepsilon}^{l}(\Omega)$ and $\mathcal{R}_{\beta}^{l}(\Omega ; \partial \Omega) \subset \mathcal{R}_{\beta-\varepsilon}^{l}(\Omega$; $\partial \Omega$ ) for all $\varepsilon>0$, it follows that

$$
\begin{aligned}
\operatorname{ker} \mathcal{S}_{\beta}^{l} & \subset \operatorname{ker} \mathcal{S}_{\beta-\varepsilon}^{l} \\
\operatorname{coker} \mathcal{S}_{\beta}^{l} & \subset \operatorname{coker} \mathcal{S}_{\beta+\varepsilon}^{l} .
\end{aligned}
$$

Consequently, the subspaces $\operatorname{ker} \mathcal{S}_{\beta}^{l}$ and coker $\mathcal{S}_{\beta}^{l}$ are finite-dimensional for all $\beta \in \mathbb{R}$. We shall show that for $\beta \in \mathbb{Z}$ the range $\operatorname{Im} \mathcal{S}_{\beta}^{l}$ is not closed and hence $\mathcal{S}_{\beta}^{l}$ looses the Fredholm property.

Let $\beta=-m-1 \quad(m \in \mathbb{Z})$. Denote by $\chi$ the smooth cut-off function with $\chi(r)=1$ for $r<1$ and $\chi(r)=0$ for $r>2$ and let $\chi_{R}(r)=\chi\left(\frac{r}{R}\right) \quad(R \geq 2)$. We take

$$
\begin{aligned}
p_{0}(y) & =-(2 \pi)^{-1} \ln r \\
p_{m}(y) & =(2 \pi|m|)^{-\frac{1}{2}} r^{m} \cos (m \varphi) \quad(m \neq 0) \\
\mathbf{u}_{m}(y, z) & =\frac{1}{2 \nu} z(z-1) \nabla p_{m}(y)
\end{aligned}
$$


and put

$$
\left(\widehat{\mathbf{u}}_{m}, \widehat{p}_{m}\right)=(1-\chi(r)) \chi_{R}(r)\left(\mathbf{u}_{m}, p_{m}\right) .
$$

It is easy to compute that

$$
\begin{aligned}
& \left\|\left(\widehat{\mathbf{u}}_{m}, \widehat{p}_{m}\right) ; \mathcal{D}_{-m-1}^{l}(\Omega)\right\|^{2} \\
& \quad \geq\left\|\left(\widehat{\mathbf{u}}_{m}, \widehat{p}_{m}\right) ; L_{-m}^{2}(\Omega)^{3} \times L_{-m-1}^{2}(\Omega)\right\|^{2} \\
& \quad \geq c\left(1+\int_{2}^{R}\left(r^{-2 m} r^{2(m-1)}+r^{-2(m+1)} r^{2 m}\right) r d r\right) \\
& \quad \geq c\left(1+\ln \frac{R}{2}\right) .
\end{aligned}
$$

On the other hand, $\left(\mathbf{u}_{m}, p_{m}\right)$ satisfies the homogeneous Stokes problem (1.2) - (1.3) in $\Omega \backslash\{x: r=0\}$. Therefore

$$
\begin{aligned}
-\nu \Delta \widehat{\mathbf{u}}_{m}+\nabla \widehat{p}_{m} & =\left[-\nu \Delta+\nabla,(1-\chi) \chi_{R}\right]\left(\mathbf{u}_{m}, p_{m}\right) \equiv \mathbf{f}_{m} & & (x \in \Omega) \\
\operatorname{div} \widehat{\mathbf{u}}_{m} & =\left[\operatorname{div},(1-\chi) \chi_{R}\right] \mathbf{u}_{m} \equiv g_{m} & & (x \in \Omega) \\
\widehat{\mathbf{u}}_{m} & =0 & & (x \in \partial \Omega)
\end{aligned}
$$

where $[A, B]$ stands for the commutator of the operators $A$ and $B$. The right-hand side $\left(\mathbf{f}_{m}, g_{m}\right)$ has a compact support lying in the union of the annuli $\{x \in \Omega: 1<r<2\}$ and $\{x \in \Omega: R<r<2 R\}$. Calculating the norm $\left\|\left(\mathbf{f}_{m}, g_{m}\right) ; \mathcal{R}_{-m-1}^{l}(\Omega ; \partial \Omega)\right\|^{2}$, we find that it is bounded by the expression

$$
c\left(1+\int_{R}^{2 R} R^{-2} r^{-2 m} r^{2 m} r d r\right) \leq \mathrm{const}
$$

where $c$ is independent of $R \in(2, \infty)$. The range $\operatorname{Im} \mathcal{S}_{-m-1}^{l}$ is closed if and only if for every $(\mathbf{v}, q) \in \mathcal{D}_{-m-1}^{l}(\Omega) \ominus \operatorname{ker} \mathcal{S}_{-m-1}^{l}$ the estimate

$$
\left\|(\mathbf{v}, q) ; \mathcal{D}_{-m-1}^{l}(\Omega)\right\| \leq c_{*}\left\|\mathcal{S}_{-m-1}^{l}(\mathbf{v}, q) ; \mathcal{R}_{-m-1}^{l}(\Omega ; \partial \Omega)\right\|
$$

holds true with constant $c_{*}$ independent of $(\mathbf{v}, q)$. Letting $R \rightarrow \infty$ in formulae (3.14) and (3.13) we see that for $\left(\widehat{\mathbf{u}}_{m}, \widehat{p}_{m}\right)$ the last estimate fails, i.e. $\operatorname{Im} \mathcal{S}_{-m-1}^{l}$ is not closed. The theorem is proved

Lemma 3.1. If $\beta \geq-1$, then $\mathcal{S}_{\beta}^{l}$ is a monomorphism, and if $\beta<-1$, then $\mathcal{S}_{\beta}^{l}$ is an epimorphism.

Proof. Let $\beta \geq-1$ and $(\mathbf{u}, p) \in \operatorname{ker} \mathcal{S}_{\beta}^{l}$. Multiplying the homogeneous equations (1.2) by $\mathbf{u}$ and integrating by parts in $\Omega$, we derive

$$
\nu \int_{\Omega}|\nabla \mathbf{u}(x)|^{2} d x=0 .
$$

(Note that by definition of the space $\mathcal{D}_{\beta}^{l}(\Omega)$ all the integrals involved converge for $\beta \geq-1$.) From (3.15) it follows $|\nabla \mathbf{u}(x)|=0$ and hence $\mathbf{u}(x)=0$. The Stokes equations (1.2) imply $\nabla p=0$ in $\Omega$, i.e. $p(x)=c$. If $c \neq 0$, then the integral $\int_{\Omega}\left(1+r^{2}\right)^{\beta}|c|^{2} d x$ diverges (recall that $\beta \geq-1$ ) what contradicts with the condition $p \in L_{\beta}^{2}(\Omega)$. Thus $c=0$ and $\operatorname{ker} \mathcal{S}_{\beta}^{l}=\emptyset$ for $\beta \geq-1$. For $\beta<-1$ the relation $\operatorname{dim}$ coker $\mathcal{S}_{\beta}^{l}=0$ follows from (3.12), since in this case $-2-\beta>-1$ and $\operatorname{ker} \mathcal{S}_{-2-\beta}^{l}=\emptyset$ 


\section{Coefficients in the asymptotics and computation of the index}

Let $(\mathbf{u}, p) \in \mathcal{D}_{\beta}^{l}(\Omega) \quad(\beta>-1)$ be a solution of the Stokes problem (1.2) - (1.3) with right-hand side $(\mathbf{f}, g, \mathbf{h}) \in \mathcal{R}_{\beta+k}^{l}(\Omega ; \partial \Omega) \quad(k \in \mathbb{N})$. From Theorem 2.4 it follows that the solution $(\mathbf{u}, p)$ admits the asymptotic representation (2.30) - (2.31). On the other hand, by Lemma 3.1 we know that the operator $\mathcal{S}_{\beta}^{l}$ with $\beta>-1$ is a monomorphism, i.e. the solution is unique. Therefore, the coefficients $c_{-m}^{ \pm}(m \in \mathbb{N})$ in the asymptotic formulae (2.30) - (2.31) are uniquely determined by the right-hand side $(\mathbf{f}, g, \mathbf{h})$. In this section we find integral formulae for the coefficients $c_{0}^{-}$and $c_{-m}^{ \pm}(m \in \mathbb{N})$.

We start with the computation of $c_{0}^{-}$.

Lemma 4.1. Let $(\mathbf{u}, p) \in \mathcal{D}_{\beta}^{l}(\Omega), \beta \in(-2,-1)$, be a solution of problem $(1.2)-(1.3)$ with right-hand side $(\mathbf{f}, g, \mathbf{h}) \in \mathcal{R}_{\beta+1}^{l}(\Omega ; \partial \Omega)$. Then the coefficient $c_{0}^{-}$in the asymptotic formula

$$
\left(\begin{array}{c}
\mathbf{u}(x) \\
p(x)
\end{array}\right)=\chi(r)\left(\begin{array}{c}
c_{0}^{+} \mathbf{u}_{0}^{+}(y, z)+c_{0}^{-} \mathbf{u}_{0}^{-}(y, z) \\
c_{0}^{+} p_{0}^{+}(y)+c_{0}^{-} p_{0}^{-}(y)
\end{array}\right)+\left(\begin{array}{c}
\tilde{\mathbf{u}}(x) \\
\tilde{p}(x)
\end{array}\right)
$$

where $(\tilde{\mathbf{u}}, \tilde{p}) \in \mathcal{D}_{\beta+1}^{l}(\Omega)$ (see (2.30)) admits the integral representations

$$
c_{0}^{-}=-12 \nu\left(\int_{\partial \Omega} \mathbf{h} \cdot \mathbf{n} d s-\int_{\Omega} g d x\right) .
$$

Proof. Let us apply to the solutions $(\mathbf{u}, p)$ and $\left(\mathbf{u}_{0}^{+}, p_{0}^{+}\right)=(\mathbf{0}, 1)$ Green's formula in the domain $\Omega_{R}=\{x \in \Omega: r<R(R>2)\}$ :

$$
\int_{\Omega_{R}}(-\nu \Delta \mathbf{u}+\nabla p) \cdot \mathbf{0} d x-\int_{\Omega_{R}} \operatorname{div} \mathbf{u} d x+\int_{\partial \Omega_{R} \cup S_{R}} \mathbf{u} \cdot \mathbf{n} d s=0
$$

where $\partial \Omega_{R}=\partial \Omega \cap \Omega_{R}$ and $S_{R}=\{x \in \Omega: r=R\}$. This furnishes

$$
-\int_{\Omega_{R}} g d x+\int_{\partial \Omega_{R}} \mathbf{h} \cdot \mathbf{n} d s+\int_{S_{R}} \mathbf{u} \cdot \mathbf{n} d s=0 .
$$

Taking into account representation (4.1) for $\mathbf{u}$, we compute

$$
\begin{aligned}
\int_{S_{R}} \mathbf{u} \cdot \mathbf{n} d s & =c_{0}^{-} \int_{S_{R}} \mathbf{u}_{0}^{-} \cdot \mathbf{n} d s+\int_{S_{R}} \tilde{\mathbf{u}} \cdot \mathbf{n} d s \\
& =-\frac{c_{0}^{-}}{4 \nu \pi} \int_{S_{R}} z(z-1) \nabla \ln r \cdot \nabla r d s+\int_{S_{R}} \tilde{\mathbf{u}} \cdot \mathbf{n} d s \\
& =\frac{c_{0}^{-}}{12 \nu}+\int_{S_{R}} \tilde{\mathbf{u}} \cdot \mathbf{n} d s .
\end{aligned}
$$

Since $\tilde{\mathbf{u}} \in L_{\beta+2}^{2}(\Omega), \beta \in(-2,-1)$, we get

$$
\begin{aligned}
\left|\int_{S_{R}} \tilde{\mathbf{u}} \cdot \mathbf{n} d s\right| & \leq c\left(R^{-2(\beta+2)+1} \int_{S_{R}}(1+r)^{2(\beta+2)}|\tilde{\mathbf{u}}|^{2} d s\right)^{\frac{1}{2}} \\
& \leq c\left(R \int_{S_{R}}(1+r)^{2(\beta+2)}|\tilde{\mathbf{u}}|^{2} d s\right)^{\frac{1}{2}} \\
& =o\left(R^{-1}\right) \rightarrow 0 \quad \text { as } R \rightarrow \infty
\end{aligned}
$$

(at least for some subsequence $R_{l}$ ). Substituting the last two formulae into (4.3) and passing to the limit as $R_{l} \rightarrow \infty$, we derive (4.2) 
In the previous lemma we have already used a special solution of the homogeneous Stokes problem $\boldsymbol{\zeta}_{0}^{+}(x)=\left(\mathbf{u}_{0}^{+}(y, z), p_{0}^{+}(y)\right)^{T}=(\mathbf{0}, 1)^{T}$. Let us construct special solutions $\boldsymbol{\zeta}_{m}^{ \pm}=\left(\boldsymbol{\xi}_{m}^{ \pm}, \eta_{m}^{ \pm}\right)^{T}$ for $m \in \mathbb{N}$.

Lemma 4.2. For every $m \in \mathbb{N}$ there exist solutions $\boldsymbol{\zeta}_{m}^{ \pm}$of the homogeneous Stokes problem (1.2) - (1.3) which admit the asymptotic forms

$$
\boldsymbol{\zeta}_{m}^{ \pm}=\left(\begin{array}{c}
\boldsymbol{\xi}_{m}^{ \pm}(x) \\
\eta_{m}^{ \pm}(x)
\end{array}\right)=\left(\begin{array}{c}
\mathbf{u}_{m}^{ \pm}(y, z) \\
p_{m}^{ \pm}(y)
\end{array}\right)+\left(\begin{array}{c}
\tilde{\boldsymbol{\xi}}_{m}^{ \pm}(x) \\
\tilde{\eta}_{m}^{ \pm}(x)
\end{array}\right) \quad(m \in \mathbb{N})
$$

where $\left(\mathbf{u}_{m}^{ \pm}(y, z), p_{m}^{ \pm}(y)\right)^{1)}$ are given by $(2.31)$ and $\left(\tilde{\boldsymbol{\xi}}_{m}^{ \pm}, \tilde{\eta}_{m}^{ \pm}\right) \in \mathcal{D}_{\gamma}^{l}(\Omega)$ with arbitrary $\gamma$ satisfying the relation

$$
-1<\gamma<0
$$

Proof. We shall look for the solution $\left(\boldsymbol{\xi}_{m}^{ \pm}, \eta_{m}^{ \pm}\right)$in form (4.4). Since $\left(\mathbf{u}_{m}^{ \pm}, p_{m}^{ \pm}\right)$ solve the homogeneous Stokes problem (1.2) - (1.3) in the layer $\Pi$, we obtain for $\left(\tilde{\boldsymbol{\xi}}_{m}^{ \pm}, \tilde{\eta}_{m}^{ \pm}\right)$the non-homogeneous problem $(1.2)$ - (1.3) with right-hand side $\left(\mathbf{0}, 0, \mathbf{h}_{m}^{ \pm}\right)$ where $\mathbf{h}_{m}^{ \pm}=-\left.\mathbf{u}_{m}^{ \pm}\right|_{\partial \Omega}$ has compact support contained in $\{x \in \partial \Omega:|x|<1\}$. Thus, $\left(\mathbf{0}, 0, \mathbf{h}_{m}^{ \pm}\right) \in \mathcal{R}_{\gamma}^{l}(\Omega ; \partial \Omega) \subset \mathcal{R}_{\gamma-1}^{l}(\Omega ; \partial \Omega)$. Since $(\gamma-1) \in(-2,-1)$, the operator $\mathcal{S}_{\gamma-1}^{l}$ is of Fredholm type (Theorem 3.1) and dim coker $\mathcal{S}_{\gamma-1}^{l}=0$ (Lemma 3.1). Therefore, problem (1.2) - (1.3) is solvable in $\mathcal{D}_{\gamma-1}^{l}(\Omega)$ for all right-hand sides from $\mathcal{R}_{\gamma-1}^{l}(\Omega ; \partial \Omega)$ and we find the remainder $\left(\tilde{\boldsymbol{\xi}}_{m}^{ \pm}, \tilde{\eta}_{m}^{ \pm}\right) \in \mathcal{D}_{\gamma-1}^{l}(\Omega)$. Moreover, $\left(\tilde{\boldsymbol{\xi}}_{m}^{ \pm}, \tilde{\eta}_{m}^{ \pm}\right)$admits the asymptotic representation (4.1):

$$
\left(\begin{array}{c}
\tilde{\boldsymbol{\xi}}_{m}^{ \pm}(x) \\
\tilde{\eta}_{m}^{ \pm}(x)
\end{array}\right)=\chi(r)\left(\begin{array}{c}
c_{0}^{+} \mathbf{u}_{0}^{+}(y, z)+c_{0}^{-} \mathbf{u}_{0}^{-}(y, z) \\
c_{0}^{+} p_{0}^{+}(y)+c_{0}^{-} p_{0}^{-}(y)
\end{array}\right)+\left(\begin{array}{l}
\widehat{\boldsymbol{\xi}}_{m}^{ \pm}(x) \\
\widehat{\eta}_{m}^{ \pm}(x)
\end{array}\right)
$$

with $\left(\widehat{\boldsymbol{\xi}}_{m}^{ \pm}, \widehat{\eta}_{m}^{ \pm}\right) \in \mathcal{D}_{\gamma}^{l}(\Omega)$. We normalize $\left(\tilde{\boldsymbol{\xi}}_{m}^{ \pm}, \tilde{\eta}_{m}^{ \pm}\right)$by the condition $\lim _{|x| \rightarrow \infty} \tilde{\eta}_{m}^{ \pm}(x)=0$ so that $c_{0}^{+}=0$. Since $\left.\tilde{\boldsymbol{\xi}}_{m}^{ \pm}\right|_{\partial \Omega}=-\left.\mathbf{u}_{m}^{ \pm}\right|_{\partial \Omega}$ on $\partial \Omega$, from (4.2) we get

$$
c_{0}^{-}=12 \nu \int_{\partial \Omega} \mathbf{h}_{m}^{ \pm} \cdot \mathbf{n} d s=12 \nu \int_{\Omega} \operatorname{div} \mathbf{u}_{m}^{ \pm}(y, z) d x=0 \quad(m \in \mathbb{N}) .
$$

Thus we obtain $\left(\widehat{\boldsymbol{\xi}}_{m}^{ \pm}, \widehat{\eta}_{m}^{ \pm}\right)=\left(\tilde{\boldsymbol{\xi}}_{m}^{ \pm}, \tilde{\eta}_{m}^{ \pm}\right) \in \mathcal{D}_{\gamma}^{l}(\Omega)$ and this concludes the proof of the lemma

Let us compute now the coefficients $c_{-m}^{ \pm}(m \in \mathbb{N})$.

Lemma 4.3. Let $(\mathbf{u}, p) \in \mathcal{D}_{\beta}^{l}(\Omega) \quad(\beta>-1)$ be a solution of problem $(1.2)-(1.3)$ with right-hand side $(\mathbf{f}, g, \mathbf{h}) \in \mathcal{R}_{\beta+k}^{l}(\Omega ; \partial \Omega) \quad(k \in \mathbb{N})$. Then the coefficients $c_{-m}^{ \pm}$in the asymptotic formulae (2.30) - (2.31) admit the integral representations

$$
\begin{gathered}
c_{-m}^{ \pm}=-12 \nu\left(\int_{\Omega} \mathbf{f} \cdot \boldsymbol{\xi}_{m}^{ \pm} d x-\int_{\Omega} g \eta_{m}^{ \pm} d x+\int_{\partial \Omega} \mathbf{h} \cdot\left(\eta_{m}^{ \pm} \mathbf{n}-\nu \partial_{n} \boldsymbol{\xi}_{m}^{ \pm}\right) d s\right) \\
(-\beta-k-1<-m<-\beta-1)
\end{gathered}
$$

1) Note that for $m \in \mathbb{N}$ the functions $p_{m}^{ \pm}$are harmonic polynomials and therefore $\left(\mathbf{u}_{m}^{ \pm}, p_{m}^{ \pm}\right) \in$ $C^{\infty}(\bar{\Omega})$. 
where $\left(\boldsymbol{\xi}_{m}^{ \pm}, \eta_{m}^{ \pm}\right)$are the solutions of the homogeneous problem $(1.2)-(1.3)$ constructed in Lemma 4.2.

Proof. Let us apply to $(\mathbf{u}, p)$ and $\left(\boldsymbol{\xi}_{m}^{ \pm}, \eta_{m}^{ \pm}\right)$Green's formula in the domain $\Omega_{R}=$ $\{x \in \Omega: r<R(R>2)\}:$

$$
\begin{aligned}
& \int_{\Omega_{R}}(-\nu \Delta \mathbf{u}+\nabla p) \cdot \boldsymbol{\xi}_{m}^{ \pm} d x-\int_{\Omega_{R}} \operatorname{div} \mathbf{u} \eta_{m}^{ \pm} d x+\int_{\partial \Omega_{R} \cup S_{R}} \mathbf{u} \cdot\left(\mathbf{n} \eta_{m}^{ \pm}-\nu \partial_{n} \boldsymbol{\xi}_{m}^{ \pm}\right) d s \\
& =\int_{\Omega_{R}}\left(-\nu \Delta \boldsymbol{\xi}_{m}^{ \pm}+\nabla \eta_{m}^{ \pm}\right) \cdot \mathbf{u} d x-\int_{\Omega_{R}} \operatorname{div} \boldsymbol{\xi}_{m}^{ \pm} p d x+\int_{\partial \Omega_{R} \cup S_{R}} \boldsymbol{\xi}_{m}^{ \pm} \cdot\left(\mathbf{n} p-\nu \partial_{n} \mathbf{u}\right) d s .
\end{aligned}
$$

Since $\left(\boldsymbol{\xi}_{m}^{ \pm}, \eta_{m}^{ \pm}\right)$fulfils the homogeneous equations (1.2) - (1.3), from (4.7) we derive

$$
\begin{aligned}
& \int_{\Omega_{R}} \mathbf{f} \cdot \boldsymbol{\xi}_{m}^{ \pm} d x-\int_{\Omega_{R}} g \eta_{m}^{ \pm} d x+\int_{\partial \Omega_{R}} \mathbf{h} \cdot\left(\mathbf{n} \eta_{m}^{ \pm}-\nu \partial_{n} \boldsymbol{\xi}_{m}^{ \pm}\right) d s \\
& +\int_{S_{R}} \mathbf{u} \cdot\left(\mathbf{n} \eta_{m}^{ \pm}-\nu \partial_{n} \boldsymbol{\xi}_{m}^{ \pm}\right) d s=\int_{S_{R}} \boldsymbol{\xi}_{m}^{ \pm} \cdot\left(\mathbf{n} p-\nu \partial_{n} \mathbf{u}\right) d s
\end{aligned}
$$

Let us calculate the right-hand side of (4.8). Taking account of the asymptotic representations $(2.30)-(2.31)$ and $(4.4)$ for $(\mathbf{u}, p)$ and $\left(\boldsymbol{\xi}_{m}^{ \pm}, \eta_{m}^{ \pm}\right)$, respectively, we get

$$
\begin{aligned}
\int_{S_{R}} & \boldsymbol{\xi}_{m}^{ \pm} \cdot\left(\mathbf{n} p-\nu \partial_{n} \mathbf{u}\right) d s \\
= & \int_{S_{R}} \tilde{\boldsymbol{\xi}}_{m}^{ \pm} \cdot\left(\mathbf{n} p-\nu \partial_{n} \mathbf{u}\right) d s \\
& \quad+\int_{S_{R}} \mathbf{u}_{m}^{ \pm} \cdot \sum_{-\beta-k-1<-l<-\beta-1}\left[\mathbf{n}\left(c_{-l}^{+} p_{-l}^{+}+c_{-l}^{-} p_{-l}^{-}\right)-\nu\left(c_{-l}^{+} \partial_{n} \mathbf{u}_{-l}^{+}+c_{-l}^{-} \partial_{n} \mathbf{u}_{-l}^{-}\right)\right] d s .
\end{aligned}
$$

The first integral in the right-hand side here can be majorated by

$$
\begin{aligned}
& \left(R \int_{S_{R}}\left|\tilde{\boldsymbol{\xi}}_{m}^{ \pm}\right|^{2}\left(1+r^{2}\right)^{\gamma+1} d s\right)^{\frac{1}{2}}\left(R \int_{S_{R}}|p|^{2}\left(1+r^{2}\right)^{\beta} R^{-2(\beta+\gamma+1)-2} d s+\right. \\
& \left.R \int_{S_{R}}|\mathbf{u}|^{2}\left(1+r^{2}\right)^{\beta+1} R^{-2(\beta+\gamma+1)-4} d s\right)^{\frac{1}{2}} \leq c\left(R \int_{S_{R}}\left|\tilde{\boldsymbol{\xi}}_{m}^{ \pm}\right|^{2}\left(1+r^{2}\right)^{\gamma+1} d s\right)^{\frac{1}{2}} \\
& \times\left(R \int_{S_{R}}|p|^{2}\left(1+r^{2}\right)^{\beta} d s+R^{-1} \int_{S_{R}}|\mathbf{u}|^{2}\left(1+r^{2}\right)^{\beta+1} d s\right)^{\frac{1}{2}} \cdot
\end{aligned}
$$

Since $\tilde{\boldsymbol{\xi}}_{m}^{ \pm} \in L_{\gamma+1}^{2}(\Omega), \mathbf{u} \in L_{\beta+1}^{2}(\Omega), p \in L_{\beta}^{2}(\Omega)$ (see the definition of the space $\mathcal{D}_{\beta}^{l}(\Omega)$ ), expresion (4.10) vanishes as $R \rightarrow \infty$ (at least, for some subsequence $R_{j} \rightarrow \infty$ ). Further, using the relations

$$
\begin{aligned}
\int_{0}^{2 \pi} \cos (m \varphi) \sin (|l| \varphi) d \varphi & =0 \\
\int_{0}^{2 \pi} \sin (|m| \varphi) \sin (|l| \varphi) d \varphi= & \int_{0}^{2 \pi} \cos (m \varphi) \cos (l \varphi) d \varphi=\pi \delta_{m, l}
\end{aligned}
$$


we find that

$$
\begin{aligned}
\int_{S_{R}} \mathbf{u}_{m}^{ \pm} & \cdot \sum_{-\beta-k<-l<-\beta-1}\left[\mathbf{n}\left(c_{-l}^{+} p_{-l}^{+}+c_{-l}^{-} p_{-l}^{-}\right)-\nu\left(c_{-l}^{+} \partial_{n} \mathbf{u}_{-l}^{+}+c_{-l}^{-} \partial_{n} \mathbf{u}_{-l}^{-}\right)\right] d s \\
= & \int_{S_{R}} \mathbf{u}_{m}^{ \pm} \cdot \mathbf{n}\left(c_{-m}^{+} p_{-m}^{+}+c_{-m}^{-} p_{-m}^{-}\right) d s \\
& -\nu \int_{S_{R}} \mathbf{u}_{m}^{ \pm} \cdot\left(c_{-m}^{+} \partial_{n} \mathbf{u}_{-m}^{+}+c_{-m}^{-} \partial_{n} \mathbf{u}_{-m}^{-}\right) d s \\
= & c_{-m}^{ \pm} \int_{S_{R}}(2 \nu)^{-1} z(z-1) \partial_{n} p_{m}^{ \pm} p_{-m}^{ \pm} d s+R^{-2} c(m) \\
= & -\frac{1}{24 \nu} c_{-m}^{ \pm}+o\left(R^{-1}\right) .
\end{aligned}
$$

Analogously one can compute the integral

$$
\int_{S_{R}} \mathbf{u} \cdot\left(\mathbf{n} \eta_{m}^{ \pm}-\nu \partial_{n} \boldsymbol{\xi}_{m}^{ \pm}\right) d s=\frac{1}{24 \nu} c_{-m}^{ \pm}+o\left(R^{-1}\right) .
$$

Substituting formulae (4.9) - (4.12) into (4.8) and passing $R \rightarrow \infty$, we derive formula (4.6)

Now we are in a position to compute the dimensions of $\operatorname{ker} \mathcal{S}_{\beta}^{l}$ and coker $\mathcal{S}_{\beta}^{l}$.

Theorem 4.1.

(i) If $\beta \in(k-1, k) \quad(0 \leq k \in \mathbb{Z})$, then $\operatorname{dim}$ coker $\mathcal{S}_{\beta}^{l}=2 k+1$.

(ii) If $\beta \in(q-1, q) \quad(\mathbb{Z} \ni q \leq-1)$, then $\operatorname{dim} \operatorname{ker} \mathcal{S}_{\beta}^{l}=-2 q-1$.

(iii) If $\beta \in(p, p+1) \quad(p \in \mathbb{Z})$, then Ind $\mathcal{S}_{\beta}^{l}=-2 p-1$.

Proof. Let $(\mathbf{f}, g, \mathbf{h}) \in \mathcal{R}_{\beta}^{l}(\Omega ; \partial \Omega) \quad(\beta \in(k-1, k), k \geq 0)$. Then there exists a solution $(\mathbf{u}, p) \in \mathcal{D}_{\beta_{1}}^{l}(\Omega) \quad\left(\beta_{1}=\beta-k-1 \in(-2,-1)\right)$ of problem (1.2) - (1.3). (Note that $\mathcal{R}_{\beta}^{l}(\Omega ; \partial \Omega) \subset \mathcal{R}_{\beta_{1}}^{l}(\Omega ; \partial \Omega)$ and by Lemma 3.1 the operator $\mathcal{S}_{\beta_{1}}^{l}\left(\beta_{1} \in(-2,-1)\right)$ is an epimorphism.) For $(\mathbf{u}, p)$ there holds the asymptotic formula (2.30) where the constants $c_{0}^{-}$and $c_{-m}^{ \pm}(m=1, \ldots, k)$ admit the integral representations (4.2) and (4.6), respectively. Hence under $2 k+1$ compatibility conditions

$$
\begin{aligned}
\int_{\partial \Omega} \mathbf{h} \cdot \mathbf{n} d s-\int_{\Omega} g d x & =0 \\
\int_{\Omega} \mathbf{f} \cdot \boldsymbol{\xi}_{m}^{ \pm} d x-\int_{\Omega} g \eta_{m}^{ \pm} d x+\int_{\partial \Omega} \mathbf{h} \cdot\left(\eta_{m}^{ \pm} \mathbf{n}-\nu \partial_{n} \boldsymbol{\xi}_{m}^{ \pm}\right) d s & =0 \quad(m=1, \ldots, k)
\end{aligned}
$$

we obtain

$$
\left(\begin{array}{c}
\mathbf{u}(x) \\
p(x)
\end{array}\right)=c_{0}^{+}\left(\begin{array}{l}
\mathbf{0} \\
1
\end{array}\right)+\left(\begin{array}{c}
\tilde{\mathbf{u}}(x) \\
\tilde{p}(x)
\end{array}\right)
$$

where $(\tilde{\mathbf{u}}, \tilde{p}) \in \mathcal{D}_{\beta}^{l}(\Omega)$. Normalizing this solution by the condition $\lim _{|x| \rightarrow \infty} p(x)=0$ we get $(\mathbf{u}, p)=(\tilde{\mathbf{u}}, \tilde{p}) \in \mathcal{D}_{\beta}^{l}(\Omega)$. Thus assuming $2 k+1$ compatibility conditions to be valid, we have proved the existence of the solution $(\mathbf{u}, p) \in \mathcal{D}_{\beta}^{l}(\Omega)$. Since for $\beta \in$ 
$(k-1, k)(k \geq 0)$ the operator $\mathcal{S}_{\beta}^{l}$ is a Fredholm monomorphism (see Lemma 3.1), these conditions are necessary. Therefore, we conclude

$$
\operatorname{dim} \text { coker } \mathcal{S}_{\beta}^{l}=2 k+1 \text {. }
$$

Statement (ii) follows now from the fact that

$$
\operatorname{dim} \operatorname{ker} \mathcal{S}_{\beta}^{l}=\operatorname{dim} \operatorname{coker} \mathcal{S}_{-\beta-2}^{l} .
$$

Statement (iii) has become evident

\section{Asymptotic conditions at infinity}

As follows from Lemma 3.1, there is no admissible $\beta$ such that the operator $\mathcal{S}_{\beta}^{l}$ is of index zero. In order to compensate this lack we introduce function spaces with detached asymptotics and impose conditions at infinity. For $\beta<-1$ the operator $\mathcal{S}_{\beta}^{l}$ is an epimorphism, and for $\beta>-1, \mathcal{S}_{\beta}^{l}$ is a monomorphism (see Lemma 3.1). Let us take

$$
\beta_{ \pm}=-1 \pm N \pm \delta \quad\left(N \in \mathbb{N}_{0}, \delta \in(0,1)\right) .
$$

For simplicity we fix the regularity index $l$ and omit it in notations. Moreover, we denote

$$
\mathcal{S}_{\beta_{ \pm}}^{l}=\mathcal{S}_{ \pm}, \quad \mathcal{D}_{\beta_{ \pm}}^{l}(\Omega)=\mathcal{D}_{ \pm}(\Omega), \quad \mathcal{R}_{\beta_{ \pm}}^{l}(\Omega ; \partial \Omega)=\mathcal{R}_{ \pm}(\Omega ; \partial \Omega) .
$$

Let us consider the mapping $\mathcal{S}_{-}: \mathcal{D}_{-}(\Omega) \longmapsto \mathcal{R}_{-}(\Omega ; \partial \Omega)$ and its preimage $\mathbb{D}_{ \pm}(\Omega)$ of the lineal $\mathcal{R}_{+}(\Omega ; \partial \Omega) \subset \mathcal{R}_{-}(\Omega ; \partial \Omega)$ (since the preimage is related both to the indices $"+"$ and " - ", we mark it by " $\pm ")$. Due to Theorem $2.4, \mathbb{D}_{ \pm}(\Omega)$ consists of vector functions $\mathbf{U}=(\mathbf{u}, p)$ taking the asymptotic form

$$
\mathbf{U}=\left(\begin{array}{c}
\mathbf{u} \\
p
\end{array}\right)=\sum_{-N \leq m \leq N} \chi\left[c_{m}^{+}\left(\begin{array}{c}
\mathbf{u}_{m}^{+} \\
p_{m}^{+}
\end{array}\right)+c_{m}^{-}\left(\begin{array}{c}
\mathbf{u}_{m}^{-} \\
p_{m}^{-}
\end{array}\right)\right]+\left(\begin{array}{c}
\tilde{\mathbf{u}} \\
\tilde{p}
\end{array}\right)
$$

where $\tilde{\mathbf{U}}=(\tilde{\mathbf{u}}, \tilde{p}) \in \mathcal{D}_{+}(\Omega)$ and $\left(\mathbf{u}_{m}^{ \pm}, p_{m}^{ \pm}\right)$are given by $(2.31)$. This means that $\mathbb{D}_{ \pm}(\Omega)$ is formed by the sum of linear combinations of the special solutions $\left(\mathbf{u}_{m}^{ \pm}, p_{m}^{ \pm}\right)$and the "rapidly" decaying remainder $\tilde{\mathbf{U}}=(\tilde{\mathbf{u}}, \tilde{p}) \in \mathcal{D}_{+}(\Omega)$. Furthermore, the quotient space $\mathbb{D}_{ \pm}(\Omega) / \mathcal{D}_{+}(\Omega)$ can be identified with $\mathbb{R}^{4 N+2}$ and we introduce in $\mathbb{D}_{ \pm}(\Omega)$ the norm induced by the asymptotic representation (5.2)

$$
\left\|\mathbf{U} ; \mathbb{D}_{ \pm}(\Omega)\right\|=\left(\left\|\tilde{\mathbf{U}} ; \mathcal{D}_{+}(\Omega)\right\|^{2}+\left\|\mathbf{a} ; \mathbb{R}^{2 N+1}\right\|^{2}+\left\|\mathbf{b} ; \mathbb{R}^{2 N+1}\right\|^{2}\right)^{\frac{1}{2}}
$$

where $\mathbf{a}$ and $\mathbf{b}$ are columns of height $2 N+1$,

$$
\begin{aligned}
& \mathbf{a}=\left(c_{0}^{-}, c_{-1}^{+}, c_{-1}^{-}, \ldots, c_{-N}^{+}, c_{-N}^{-}\right)^{T} \\
& \mathbf{b}=\left(c_{0}^{+}, c_{1}^{+}, c_{1}^{-}, \ldots, c_{N}^{+}, c_{N}^{-}\right)^{T} .
\end{aligned}
$$

Let $\mathfrak{S}_{ \pm}$be the restriction of $\mathcal{S}_{-}$on $\mathbb{D}_{ \pm}(\Omega)$. Due to estimate (2.32),

$$
\left\|\mathbf{a} ; \mathbb{R}^{2 N+1}\right\|+\left\|\mathbf{b} ; \mathbb{R}^{2 N+1}\right\| \leq c\left(\left\|\mathfrak{S}_{ \pm} \mathbf{U} ; \mathcal{R}_{+}(\Omega ; \partial \Omega)\right\|+\left\|(\mathbf{u}, p) ; L_{\beta_{-}}^{2}(\Omega)\right\|\right) .
$$

Therefore the operator

$$
\mathfrak{S}_{ \pm}: \mathbb{D}_{ \pm}(\Omega) \longmapsto \mathcal{R}_{+}(\Omega ; \partial \Omega)
$$

of problem (1.2) - (1.3) is continuous. Moreover, in view of Theorems 3.1 and 4.1, it inherits properties of $\mathcal{S}_{-}$and the following assertion is valid. 
Theorem 5.1. The mapping (5.4) is a Fredholm epimorphism and

$$
\operatorname{dim} \operatorname{ker} \mathfrak{S}_{ \pm}=\operatorname{dim} \operatorname{ker} \mathcal{S}_{-}=2 N+1
$$

There appear the continuous projections

$$
\begin{aligned}
& \mathbb{D}_{ \pm}(\Omega) \ni \mathbf{U} \longmapsto \pi_{1} \mathbf{U}=\mathbf{a} \in \mathbb{R}^{2 N+1} \\
& \mathbb{D}_{ \pm}(\Omega) \ni \mathbf{U} \longmapsto \pi_{0} \mathbf{U}=\mathbf{b} \in \mathbb{R}^{2 N+1} .
\end{aligned}
$$

We also determine

$$
\pi=\left(\begin{array}{c}
\pi_{1} \\
\pi_{0}
\end{array}\right): \mathbb{D}_{ \pm}(\Omega) \longmapsto \mathbb{R}^{4 N+2}
$$

We treat $\pi_{0} \mathbf{U}, \pi_{1} \mathbf{U}$ and $\pi \mathbf{U}$ as columns in $\mathbb{R}^{2 N+1}, \mathbb{R}^{2 N+1}$ and $\mathbb{R}^{4 N+2}$, respectively.

Let us connect with Green's formula (2.33) the linear form

$$
Q_{\Omega}(\mathbf{U}, \mathbf{V})=Q_{\Omega}(\mathbf{u}, p ; \mathbf{v}, q)
$$

defined by

$$
\begin{aligned}
Q_{\Omega}(\mathbf{U} ; \mathbf{V}) \equiv & (-\nu \Delta \mathbf{u}+\nabla p, \mathbf{v})_{\Omega}+(-\operatorname{div} \mathbf{u}, q)_{\Omega}+\left(\mathbf{u}, q \mathbf{n}-\nu \partial_{n} \mathbf{v}\right)_{\partial \Omega} \\
& -(\mathbf{u},-\nu \Delta \mathbf{v}+\nabla q)_{\Omega}-(p,-\operatorname{div} \mathbf{v})_{\Omega}-\left(p \mathbf{n}-\nu \partial_{n} \mathbf{u}, \mathbf{v}\right)_{\partial \Omega}
\end{aligned}
$$

where $(\cdot, \cdot)_{\Omega}$ and $(\cdot, \cdot)_{\partial \Omega}$ stand for extensions of the scalar products in $L^{2}(\Omega)$ and $L^{2}(\partial \Omega)$, respectively. Since $\left(\mathbf{u}_{m}^{ \pm}, p_{m}^{ \pm}\right)$satisfy the homogeneous equations (1.2) - (1.3) in $\Pi \backslash\{x \in$ $\left.\mathbb{R}^{3}: r=0\right\}$, for any $\mathbf{U}, \mathbf{V} \in \mathbb{D}_{ \pm}(\Omega)$ we get the inclusions (see (5.2))

$$
\left.\begin{array}{l}
\left(-\nu \Delta \mathbf{u}+\nabla p,-\operatorname{div} \mathbf{u},\left.\mathbf{u}\right|_{\partial \Omega}\right) \\
\left(-\nu \Delta \mathbf{v}+\nabla q,-\operatorname{div} \mathbf{v},\left.\mathbf{v}\right|_{\partial \Omega}\right)
\end{array}\right\} \in \mathcal{R}_{+}(\Omega, \partial \Omega)
$$

and therefore all integrals in the left-hand side of (5.7) converge. Hence $Q_{\Omega}$ is a continuous antisymmetric form on $\mathbb{D}_{ \pm}(\Omega)^{2}$,

$$
Q_{\Omega}(\mathbf{V} ; \mathbf{U})=-Q_{\Omega}(\mathbf{U} ; \mathbf{V})
$$

Due to Lemma 2.7,

$$
Q_{\Omega}(\mathbf{V} ; \mathbf{U})=Q_{\Omega}(\mathbf{U} ; \mathbf{V})=0
$$

for all $\mathbf{V} \in \mathbb{D}_{+}(\Omega) \subset \mathbb{D}_{ \pm}(\Omega)$ and all $\mathbf{U} \in \mathbb{D}_{ \pm}(\Omega)$. Thus $Q_{\Omega}$ can be naturally treated as a form defined on the quotient space

$$
\left(\mathbb{D}_{ \pm}(\Omega) / \mathcal{D}_{+}(\Omega)\right)^{2} \approx \mathbb{R}^{4 N+2} \times \mathbb{R}^{4 N+2}
$$

Lemma 5.1. If $\mathbf{U}, \mathbf{V} \in \mathbb{D}_{ \pm}(\Omega)$, then

$$
Q_{\Omega}(\mathbf{U} ; \mathbf{V})=\left\langle\pi_{0} \mathbf{U}, \pi_{1} \mathbf{V}\right\rangle_{2 N+1}-\left\langle\pi_{1} \mathbf{U}, \pi_{0} \mathbf{V}\right\rangle_{2 N+1}
$$

where $\langle\cdot, \cdot\rangle_{K}=12 \nu[\cdot, \cdot]_{K}$ with $[\cdot, \cdot]_{K}$ being the scalar product in $\mathbb{R}^{K}$. 
Proof. According to the asymptotic form (5.2), we can represent $\mathbf{U}$ as sum

$$
\begin{aligned}
\mathbf{U}=\left(\begin{array}{c}
\mathbf{u} \\
p
\end{array}\right)= & \sum_{1 \leq m \leq N} \chi\left[c_{0}^{+}\left(\begin{array}{c}
\mathbf{u}_{0}^{+} \\
p_{0}^{+}
\end{array}\right)+c_{m}^{+}\left(\begin{array}{c}
\mathbf{u}_{m}^{+} \\
p_{m}^{+}
\end{array}\right)+c_{m}^{-}\left(\begin{array}{c}
\mathbf{u}_{m}^{-} \\
p_{m}^{-}
\end{array}\right)\right] \\
& +\sum_{-N \leq m \leq-1} \chi\left[c_{0}^{-}\left(\begin{array}{c}
\mathbf{u}_{0}^{-} \\
p_{0}^{-}
\end{array}\right)+c_{m}^{+}\left(\begin{array}{c}
\mathbf{u}_{m}^{+} \\
p_{m}^{+}
\end{array}\right)+c_{m}^{-}\left(\begin{array}{c}
\mathbf{u}_{m}^{-} \\
p_{m}^{-}
\end{array}\right)\right]+\left(\begin{array}{c}
\tilde{\mathbf{u}} \\
\tilde{p}
\end{array}\right) \\
= & \mathbf{U}_{N}+\mathbf{U}_{-N}+\widetilde{\mathbf{U}}\left(\widetilde{\mathbf{U}} \in \mathcal{D}_{+}(\Omega)\right) .
\end{aligned}
$$

Analogously,

$$
\mathbf{V}=\mathbf{V}_{N}+\mathbf{V}_{-N}+\widetilde{\mathbf{V}} \quad\left(\tilde{\mathbf{V}} \in \mathcal{D}_{+}(\Omega)\right)
$$

By virtue of $(5.9), Q_{\Omega}(\mathbf{U}, \tilde{\mathbf{V}})=Q_{\Omega}(\widetilde{\mathbf{U}}, \mathbf{V})=0$ so that

$$
\begin{aligned}
Q_{\Omega}(\mathbf{U}, \mathbf{V})-Q_{\Omega}\left(\mathbf{U}_{-N}, \mathbf{V}_{N}\right) & -Q_{\Omega}\left(\mathbf{U}_{N}, \mathbf{V}_{-N}\right)-Q_{\Omega}\left(\mathbf{U}_{-N}, \mathbf{V}_{-N}\right) \\
& =Q_{\Omega}\left(\mathbf{U}_{N}, \mathbf{V}_{N}\right) .
\end{aligned}
$$

Arguing as in the proof of Lemmata 4.1 and 4.3 and applying Green's formula in the truncated domain $\Omega_{R}$, we find that

$$
\begin{aligned}
\lim _{R \rightarrow \infty}\left(Q_{\Omega_{R}}\left(\mathbf{U}_{-N}, \mathbf{V}_{N}\right)+Q_{\Omega_{R}}\left(\mathbf{U}_{N}, \mathbf{V}_{-N}\right)\right) & =\left\langle\pi_{1} \mathbf{U}, \pi_{0} \mathbf{V}\right\rangle_{2 N+1}-\left\langle\pi_{0} \mathbf{U}, \pi_{1} \mathbf{V}\right\rangle_{2 N+1} \\
\lim _{R \rightarrow \infty} Q_{\Omega_{R}}\left(\mathbf{U}_{-N}, \mathbf{V}_{-N}\right) & =0 .
\end{aligned}
$$

Thus, the left-hand side of equality (5.11) is finite. The term $Q_{\Omega_{R}}\left(\mathbf{U}_{N}, \mathbf{V}_{N}\right)$ is equal to the sum $\sum_{j=1}^{2 N} \alpha_{j} R^{j}$ where $\alpha_{j}$ are constants. Therefore, its limit as $R \rightarrow \infty$ can be finite only if $\alpha_{j}=0 \quad(j=1, \ldots, 2 N$; arguing as in the proof of Lemma 4.3, one can compute directly that $\left.\alpha_{j}=0\right)$. Thus, we have got the equality $Q_{\Omega}\left(\mathbf{U}_{N}, \mathbf{V}_{N}\right)=0$ which together with (5.11) - (5.12) implies (5.10)

- We call (5.10) the generalized Green's formula.

Lemma 5.2. Let

$$
\mathbb{X}=\left(\begin{array}{l}
\mathbb{B} \\
\mathbb{S}
\end{array}\right) \quad \text { and } \quad \mathbb{Y}=\left(\begin{array}{c}
-\mathbb{T} \\
\mathbb{Q}
\end{array}\right)
$$

where $\mathbb{B}, \mathbb{T}, \mathbb{S}, \mathbb{Q}$ are $(2 N+1) \times(4 N+2)$-matrices. Suppose that $\mathbb{X}$ and $\mathbb{Y}$ satisfy the relation

$$
\mathbb{Y}^{*} \mathbb{X}=\mathbb{J} \equiv\left(\begin{array}{cc}
\mathbb{O} & \mathbb{I} \\
-\mathbb{I} & \mathbb{O}
\end{array}\right) .
$$

Then the generalized Green's formula (5.10) may be rewritten as

$$
\begin{aligned}
& (-\nu \Delta \mathbf{u}+\nabla p, \mathbf{v})_{\Omega}+(-\operatorname{div} \mathbf{u}, q)_{\Omega}+(\mathbf{u}, T \mathbf{V})_{\partial \Omega}+\langle\mathbb{B} \pi \mathbf{U}, \mathbb{T} \pi \mathbf{V}\rangle_{2 N+1} \\
& \quad=(\mathbf{u},-\nu \Delta \mathbf{v}+\nabla q)_{\Omega}+(p,-\operatorname{div} \mathbf{v})_{\Omega}+(T \mathbf{U}, \mathbf{v})_{\partial \Omega}+\langle\mathbb{S} \pi \mathbf{U}, \mathbb{Q} \pi \mathbf{V}\rangle_{2 N+1}
\end{aligned}
$$

where $T \mathbf{U}=\left.\left(p \mathbf{n}-\nu \partial_{n} \mathbf{u}\right)\right|_{\partial \Omega}$.

Proof. Simple algebraic manipulations with matrices turn (5.10) into (5.15) (cf. [12: Section 6.2.2] and [16: Lemma 6.2]) 


\section{Remark 5.1.}

1) From (5.14) it follows that $\operatorname{det} \mathbb{X} \neq 0$ and $\mathbb{Y}=\left(\mathbb{\mathbb { X }} \mathbb{X}^{-1}\right)^{*}$. Therefore, for any $(2 N+1) \times(4 N+2)$-matrix $\mathbb{B}$, the rank of which is equal to $2 N+1$, there exist matrices $\mathbb{S}, \mathbb{T}, \mathbb{Q}$ such that $(5.13)-(5.15)$ are fulfilled. If $\mathbb{S}$ is also fixed and $\operatorname{det}\left(\begin{array}{c}\mathbb{B} \\ \mathbb{S}\end{array}\right) \neq 0$, then $\mathbb{T}$ and $\mathbb{Q}$ are uniquely defined.

2) If $\mathbb{S}=\mathbb{T}$ and $\mathbb{Q}=\mathbb{B}$, Green's formula (5.15) takes the form

$$
\begin{aligned}
& (-\nu \Delta \mathbf{u}+\nabla p, \mathbf{v})_{\Omega}+(-\operatorname{div} \mathbf{u}, q)_{\Omega}+(\mathbf{u}, T \mathbf{V})_{\partial \Omega}+\langle\mathbb{B} \pi \mathbf{U}, \mathbb{T} \pi \mathbf{V}\rangle_{2 N+1} \\
& \quad=(\mathbf{u},-\nu \Delta \mathbf{v}+\nabla q)_{\Omega}+(p,-\operatorname{div} \mathbf{v})_{\Omega}+(T \mathbf{U}, \mathbf{v})_{\partial \Omega}+\langle\mathbb{T} \pi \mathbf{U}, \mathbb{B} \pi \mathbf{V}\rangle_{2 N+1}
\end{aligned}
$$

- We call (5.16) the symmetric generalized Green's formula.

Based on the generalized Green's formulae (5.15) and (5.16) and arguing in the same way as in $[12,16]$, we provide problem (1.2) - (1.3) with the additional conditions

$$
\mathbb{B} \pi \mathbf{U}=\mathbf{H} \in \mathbb{R}^{2 N+1} .
$$

- We call (5.17) the asymptotic conditions at infinity.

We connect problem (1.2) - (1.3), (5.17) with the mapping

$$
\mathbb{D}_{ \pm}(\Omega) \ni \mathbf{U} \longmapsto \mathbb{A} \mathbf{U}=\left(\mathfrak{S}_{ \pm} \mathbf{U}, \mathbb{B} \pi \mathbf{U}\right) \in \mathbb{R}_{ \pm}(\Omega ; \partial \Omega)
$$

where $\mathbb{R}_{ \pm}(\Omega ; \partial \Omega)=\mathcal{R}_{+}(\Omega ; \partial \Omega) \times \mathbb{R}^{2 N+1}$. It is clear that $\mathbb{A}$ inherits the Fredholm property from $\mathfrak{S}_{ \pm}$. Furthermore, in (5.18) we observe $2 N+1$ additional conditions and therefore the difference of the indices of $\mathfrak{S}_{ \pm}$and $\mathbb{A}$ is equal to $2 N+1$, i.e. Ind $\mathbb{A}=0$. Precisely, this equality follows from

$$
\operatorname{Ind} \mathbb{A}=\operatorname{Ind}\left(\left.\mathfrak{S}_{ \pm}\right|_{\left\{\mathbf{U} \in \mathbb{D}_{ \pm}(\Omega): \mathbb{B} \pi \mathbf{U}=0\right\}}\right)=\operatorname{Ind} \mathfrak{S}_{ \pm}-(2 N+1)=0 .
$$

\section{Theorem 5.2.}

1) $\operatorname{ker} \mathbb{A}=\left\{\mathbf{V} \in \operatorname{ker} \mathfrak{S}_{ \pm}: \mathbb{B} \pi \mathbf{V}=0\right\}$.

2) If the generalized Green's formula (5.15) is valid, then

$$
\operatorname{coker} \mathbb{A}=\left\{\left(\mathbf{V},\left.T \mathbf{V}\right|_{\partial \Omega}, \mathbb{T} \pi \mathbf{V}\right): \mathbf{V} \in \operatorname{ker} \mathfrak{S}_{ \pm}, \mathbb{Q} \pi \mathbf{V}=0\right\}
$$

Proof. The first assertion follows from the inclusion ker $\mathbb{A} \subset \operatorname{ker} \mathfrak{S}_{ \pm}$, the second one has been proved in [12: Proposition 6.2.5] (see also [16: Theorem 6.5])

The subspace dim ker $\mathfrak{S}_{ \pm}$contains the solution $\boldsymbol{\zeta}_{0}^{+}=(\mathbf{0}, 1)$ and the solutions $\boldsymbol{\zeta}_{m}^{ \pm}=$ $\left(\boldsymbol{\xi}_{m}^{ \pm}, \eta_{m}^{ \pm}\right) \quad(m=1, \ldots, N)$ of the homogeneous problem (1.2) - (1.3) (see Lemma 4.2). Since the dimension of ker $\mathfrak{S}_{ \pm}$coincides with the number of linear independent solutions we have found that ker $\mathfrak{S}_{ \pm}$becomes the linear hull of them:

$$
\operatorname{ker} \mathfrak{S}_{ \pm}=\mathcal{L}\left\{\boldsymbol{\zeta}_{0}^{+}, \boldsymbol{\zeta}_{1}^{+}, \boldsymbol{\zeta}_{1}^{-}, \ldots, \boldsymbol{\zeta}_{N}^{+}, \boldsymbol{\zeta}_{N}^{-}\right\} \equiv\left\{\boldsymbol{\zeta}=\mathfrak{Z} \mathbf{c}: \mathbf{c} \in \mathbb{R}^{2 N+1}\right\}
$$

where $\mathfrak{Z}=\left(\boldsymbol{\zeta}_{0}^{+}, \boldsymbol{\zeta}_{1}^{+}, \boldsymbol{\zeta}_{1}^{-}, \ldots, \boldsymbol{\zeta}_{N}^{+}, \boldsymbol{\zeta}_{N}^{-}\right)$is a $4 \times(2 N+1)$-matrix-function or, what is the same, a row of solutions. Due to Lemma 4.2 , each element $\boldsymbol{\zeta} \in \operatorname{ker} \mathfrak{S}_{ \pm}$can be represented in the form

$$
\zeta=\mathfrak{Z c}=\mathfrak{X} \mathbf{c}-\chi \mathfrak{Y} \mathfrak{M} \mathbf{c}+\widetilde{\mathfrak{U}} \mathbf{c}
$$


where the solution rows $\mathfrak{X}$ and $\mathfrak{Y}$ are defined by

$$
\begin{aligned}
& \mathfrak{X}=\left(\left(\begin{array}{c}
\mathbf{u}_{0}^{+} \\
p_{0}^{+}
\end{array}\right),\left(\begin{array}{c}
\mathbf{u}_{1}^{+} \\
p_{1}^{+}
\end{array}\right),\left(\begin{array}{l}
\mathbf{u}_{1}^{-} \\
p_{1}^{-}
\end{array}\right), \ldots,\left(\begin{array}{c}
\mathbf{u}_{N}^{+} \\
p_{N}^{+}
\end{array}\right),\left(\begin{array}{l}
\mathbf{u}_{N}^{-} \\
p_{N}^{-}
\end{array}\right)\right) \\
& \mathfrak{Y}=\left(\left(\begin{array}{l}
\mathbf{u}_{0}^{-} \\
p_{0}^{-}
\end{array}\right),\left(\begin{array}{l}
\mathbf{u}_{-1}^{+} \\
p_{-1}^{+}
\end{array}\right),\left(\begin{array}{l}
\mathbf{u}_{-1}^{-} \\
p_{-1}^{-}
\end{array}\right), \ldots,\left(\begin{array}{l}
\mathbf{u}_{-N}^{+} \\
p_{-N}^{+}
\end{array}\right),\left(\begin{array}{l}
\mathbf{u}_{-N}^{-} \\
p_{-N}^{-}
\end{array}\right)\right),
\end{aligned}
$$

$\mathfrak{M}$ is a constant $(2 N+1) \times(2 N+1)$-matrix and $\widetilde{\mathfrak{U}} \in \mathcal{D}_{+}(\Omega)^{2 N+1}$. Note that

$$
\left.\begin{array}{l}
\pi_{0} \mathfrak{Z} \mathbf{c}=\mathbf{c} \\
\pi_{1} \mathfrak{Z} \mathbf{c}=-\mathfrak{M c}
\end{array}\right\} .
$$

- We call the matrix $\mathfrak{M}$ the augmented flow polarization matrix.

Theorem 5.3. $\mathfrak{M}$ is a symmetric matrix.

Proof. Let $\mathbf{c}, \mathbf{C}$ be arbitrary constant vectors in $\mathbb{R}^{2 N+1}$. Since $\mathfrak{Z} \mathbf{c}$ and $\mathfrak{Z C}$ are solutions of the homogeneous problem $(1.2)-(1.3)$ we get $Q_{\Omega}(\mathfrak{Z} \mathbf{c} ; \mathfrak{Z} \mathbf{C})=0$. On the other hand, from the generalized Green's formula (5.10) there follows that

$$
\begin{aligned}
Q_{\Omega}(\mathfrak{Z} \mathbf{c} ; \mathfrak{Z C}) & =\left\langle\pi_{0} \mathfrak{Z} \mathbf{c}, \pi_{1} \mathfrak{Z C}\right\rangle_{2 N+1}-\left\langle\pi_{1} \mathfrak{Z} \mathbf{c}, \pi_{0} \mathfrak{Z} \mathbf{C}\right\rangle_{2 N+1} \\
& =\langle\mathfrak{M} \mathbf{c}, \mathbf{C}\rangle_{2 N+1}-\langle\mathbf{c}, \mathfrak{M C}\rangle_{2 N+1} \\
& =\left\langle\mathbf{c},\left(\mathfrak{M}^{*}-\mathfrak{M}\right) \mathbf{C}\right\rangle_{2 N+1} \\
& =0 .
\end{aligned}
$$

Thus, $\mathfrak{M}=\mathfrak{M}^{*}$

Remark 5.2. The matrix $\mathfrak{M}$ has the form $\mathfrak{M}=\left(\begin{array}{cc}0 & \mathbf{0} \\ \mathbf{0}^{T} & \mathbb{M}\end{array}\right)$ where $\mathbf{0}=(0, \ldots, 0)$ and $\mathbb{M}$ is a symmetric $2 N \times 2 N$-matrix. This follows from the fact that the solution $\boldsymbol{\zeta}_{0}^{+}$has the form $\boldsymbol{\zeta}_{0}^{+}=(\mathbf{0}, 1)^{T}$ and from the symmetry of $\mathfrak{M}$.

- We call the matrix $\mathbb{M}$ the flow polarization matrix.

Theorem 5.4. Let $\mathfrak{B}=\mathbb{B}(-\mathfrak{M}, \mathbb{I})^{T}$ where $\mathbb{I}$ is the unit $(2 N+1) \times(2 N+1)$-matrix. Then

$$
\operatorname{dim} \operatorname{ker} \mathbb{A}=2 N+1-\operatorname{rank} \mathfrak{B} .
$$

Proof. The elements $\boldsymbol{\zeta} \in \operatorname{ker} \mathfrak{S}_{ \pm}$admit the representation $\boldsymbol{\zeta}=\mathfrak{Z} \mathbf{c} \quad\left(\mathbf{c} \in \mathbb{R}^{2 N+1}\right.$; see (5.21)). Since $\pi_{1} \boldsymbol{\zeta}=\mathbf{c}, \pi_{0} \boldsymbol{\zeta}=-\mathfrak{M c}$ and due to the symmetry of $\mathfrak{M}, \mathbb{B} \pi \boldsymbol{\zeta}=0$ if and only if $\mathbb{B}(-\mathfrak{M}, \mathbb{I})^{T} \mathbf{c}=0$. Therefore, owing to Theorem $5.2 /(1)$ we conclude $(5.23)$

Remark 5.3. In view of (5.19) the compatibility conditions for problem (1.2) (1.3), (5.17) take the form

$$
(\mathbf{f}, \mathbf{v})_{\Omega}+(g, q)_{\Omega}+(\mathbf{h}, T \mathbf{U})_{\partial \Omega}+\langle\mathbf{H}, \mathbb{T} \pi \mathbf{V}\rangle_{2 N+1}=0
$$

for all $\mathbf{V}=(\mathbf{v}, q) \in \operatorname{ker} \mathfrak{S}_{ \pm}$with $\mathbb{Q} \pi \mathbf{V}=0$.

In accordance with $(5.19),(5.24)$ it is very natural to say that problems (1.2) - (1.3), (5.17) and (1.2) - (1.3) with additional conditions

$$
\mathbb{Q} \pi \mathbf{V}=\mathbf{K} \in \mathbb{R}^{2 N+1}
$$

are adjoint with respect to the generalized Green's formula (5.15). In the case when the symmetric generalized Green's formula (5.16) takes place, problem (1.2) - (1.3), (5.17) becomes formally self-adjoint. 


\section{Theorem 5.5.}

1) If $\Omega=\Pi$, then $\mathbb{M}=\mathbb{O}$.

2) If $\Omega \neq \Pi$ and $\Omega \subset \Pi$, then the matrix $\mathbb{M}$ is positive definite.

Proof. Let $\mathbf{c}=\left(0, \mathbf{c}^{\prime}\right)$ with $\mathbf{c}^{\prime} \in \mathbb{R}^{2 N} \backslash\{0\}$ be arbitrary. We take

$$
\mathbf{V}=(\mathbf{v}, q)=\mathfrak{Z} \mathbf{c}=\mathbf{V}^{0}+\mathbf{V}^{\#} \in \operatorname{ker} \mathfrak{S}_{ \pm}
$$

where

$$
\begin{aligned}
\mathbf{V}^{0} & =\left(\mathbf{v}^{0}, q^{0}\right)=\mathfrak{X} \mathbf{c} \\
\mathbf{V}^{\#} & =\left(\mathbf{v}^{\#}, q^{\#}\right)=-\chi \mathfrak{Y} \mathfrak{M} \mathbf{c}+\tilde{\mathfrak{U}} \mathbf{c} \in \mathcal{D}_{\gamma}^{l}(\Omega) \quad(\gamma \in(-1,0))
\end{aligned}
$$

(see (5.21) and Lemma 4.2). By formula (4.6) and the definition of $\mathbb{M}$ we get

$$
\left\langle\mathbb{M} \mathbf{c}^{\prime}, \mathbf{c}^{\prime}\right\rangle_{2 N}=\int_{\partial \Omega} \mathbf{v}^{\#} \cdot T(\mathbf{V}) d s
$$

(Note that $-\nu \Delta \mathbf{v}^{\#}+\nabla q^{\#}=0$ and $\operatorname{div} \mathbf{v}^{\#}=0$.) If $\Omega=\Pi$, then $\mathbf{V}^{0}$ is the exact solution of the homogeneous problem (1.2) - (1.3). Hence $\mathbf{V}^{\#}=0$ and $\mathbb{M}=\mathbb{O}$.

Since $\mathbf{v}^{\#}=-\mathbf{v}^{0}$ on $\partial \Omega$,

$$
\int_{\partial \Omega} \mathbf{v}^{\#} \cdot T(\mathbf{V}) d s=\int_{\partial \Omega} \mathbf{v}^{\#} \cdot T\left(\mathbf{V}^{\#}\right) d s-\int_{\partial \Omega} \mathbf{v}^{0} \cdot T\left(\mathbf{V}^{0}\right) d s .
$$

Integrating by parts in $\Omega$ and $\Pi \backslash \Omega$, we derive

$$
\begin{aligned}
\int_{\partial \Omega} \mathbf{v}^{\#} \cdot T\left(\mathbf{V}^{\#}\right) d s & =\int_{\Omega}\left|\nabla \mathbf{v}^{\#}\right|^{2} d x \\
\int_{\partial \Omega} \mathbf{v}^{0} \cdot T\left(\mathbf{V}^{0}\right) d s & =-\int_{\Pi \backslash \Omega}\left|\nabla \mathbf{v}^{0}\right|^{2} d x .
\end{aligned}
$$

The sign " - " in the second equality of (5.28) appears because of the oposite direction of the outward normal $\mathbf{n}$. The Dirichlet integral of $\mathbf{v}^{\#}$ is finite since $\mathbf{V}^{\#} \in \mathcal{D}_{\gamma}^{l}(\Omega)$ for $\gamma \in(-1,0)$. The formula

$$
\left\langle\mathbb{M} \mathbf{c}^{\prime}, \mathbf{c}^{\prime}\right\rangle_{2 N}=\int_{\Omega}\left|\nabla \mathbf{v}^{\#}\right|^{2} d x+\int_{\Pi \backslash \Omega}\left|\nabla \mathbf{v}^{0}\right|^{2} d x>0
$$

follows from (5.26) - (5.28) and completes the proof

Example 5.1. Let $N=0$ and $\mathbb{B}=(1,0)$ is a matrix of size $1 \times 2$. Then the condition $\mathbb{B} \pi \mathbf{U}=\pi_{1} \mathbf{U}=c_{0}^{-}$prescribes the total flux of the fluid over the surface $S_{R}$. The matrix $\mathfrak{Z}$ consists of one solution $\boldsymbol{\zeta}_{0}^{+}$. Hence $\operatorname{dim}$ ker $\mathfrak{S}_{ \pm}=1, \pi_{1} \mathfrak{Z} \mathbf{c}=0$ for all $\mathbf{c}$ and $\mathfrak{M}=\mathbb{O}$ (see $(5.22)$ ). We have $\mathfrak{B}=\mathbb{B}(-\mathfrak{M}, \mathbb{I})^{T}=\mathbb{O}$ and, by Theorem $5.4, \operatorname{dim} \operatorname{ker} \mathbb{A}=1-\operatorname{rank} \mathfrak{B}=1$. Therefore the operator $\mathbb{A}$ is an epimorphism with one-dimentional kernel (constant pressure). 
If $\mathbb{B}=(0,1)$, then $\mathbb{B} \pi \mathbf{U}=\pi_{0} \mathbf{U}=c_{0}^{+}$prescribes the limit of the pressure component as $r \rightarrow \infty$. We get $\pi_{0} \mathfrak{Z} \mathbf{c}=1, \mathbb{M}=\mathbb{I}$ and $\mathfrak{B}=\mathbb{B}(-\mathfrak{M}, \mathbb{I})^{T}=\mathbb{I}$. By Theorem 5.4, $\operatorname{dim} \operatorname{ker} \mathbb{A}=1-\operatorname{rank} \mathfrak{B}=0$ and the operator $\mathbb{A}$ is an isomorphism.

Example 5.2. Let $N=1$ and

$$
\mathbb{B}=\left(\begin{array}{cccccc}
1 & 0 & 0 & 0 & 0 & 0 \\
0 & 0 & 0 & 0 & \cos \alpha & \sin \alpha \\
0 & 0 & 0 & 0 & -\sin \alpha & \cos \alpha
\end{array}\right)
$$

We consider the condition $\mathbb{B} \pi \mathbf{U}=\left(H_{1}, H_{2}, 0\right)^{T}$ which prescribes the total flux $H_{1}$ over $S_{R}$ and the linear flux $H_{2}$ of $\mathbf{u}$ in the direction $\mathbf{e}^{\alpha}=(\cos \alpha, \sin \alpha)$ (cf. [14]). We obtain $\mathfrak{Z}=\left\{\boldsymbol{\zeta}_{0}^{+}, \boldsymbol{\zeta}_{1}^{+}, \boldsymbol{\zeta}_{1}^{-}\right\}$, dim ker $\mathfrak{S}_{ \pm}=3$ and

$$
\mathfrak{B}=\mathbb{B}(-\mathfrak{M}, \mathbb{I})^{T}=\left(\begin{array}{ccc}
0 & 0 & 0 \\
0 & \cos \alpha & \sin \alpha \\
0 & -\sin \alpha & \cos \alpha
\end{array}\right)
$$

Hence $\operatorname{dim} \operatorname{ker} \mathbb{A}=3-\operatorname{rank} \mathfrak{B}=1$ and the operator $\mathbb{A}$ is an epimorphism.

If we prescribe instead of the total flux the limit $H_{1}$ of the pressure component as $r \rightarrow \infty$, we shall take

$$
\mathbb{B}=\left(\begin{array}{cccccc}
0 & 0 & 0 & 1 & 0 & 0 \\
0 & 0 & 0 & 0 & \cos \alpha & \sin \alpha \\
0 & 0 & 0 & 0 & -\sin \alpha & \cos \alpha
\end{array}\right)
$$

and consider the condition $\mathbb{B} \pi \mathbf{U}=\left(H_{1}, H_{2}, 0\right)^{T}$. In this case we get the unitary matrix

$$
\mathfrak{B}=\mathbb{B}(-\mathfrak{M}, \mathbb{I})^{T}=\left(\begin{array}{ccc}
1 & 0 & 0 \\
0 & \cos \alpha & \sin \alpha \\
0 & -\sin \alpha & \cos \alpha
\end{array}\right)
$$

$\operatorname{dim} \operatorname{ker} \mathbb{A}=3-\operatorname{rank} \mathfrak{B}=0$ and the operator $\mathbb{A}$ is an isomorphism.

\section{References}

[1] Kondratjev, V. A.: Boundary value problems for elliptic equations in domains with conical or angular points (in Russian). Trudy Moskov. Mat. Obshch. 16 (1967), 209 - 292; English transl. in: Trans. Moscow Math. Soc. 16 (1967).

[2] Kozlov, V. A., Mazja, V. G. and J. Rossmann: Elliptic Boundary Value Problems in Domains with Point Singularities (Math. Surv. \& Mon.: Vol. 52). Providence (R.I., USA): Amer. Math. Soc. 1997.

[3] Lions, J. L. and E. Magenes: Nonhomogeneous Boundary Value Problems, Vol. I. Berlin: Springer-Verlag 1972.

[4] Nazarov, S. A.: Vishik-Lyusternik method for elliptic boundary value problems in regions with conical points. Part I: The problem in a cone (in Russian). Sibirsk. Mat. Zh. 22 (1981)4, 142 - 163; Engl. transl. in: Siberian Math. J. 22 (1982), 594 - 611. 
[5] Nazarov, S. A.: Vishik-Lyusternik method for elliptic boundary value problems in regions with conical points. Part II: The problem in a bounded region (in Russian). Sibirsk. Mat. Zh. 22 (1981)5, 132 - 152; Engl. transl. in: Siberian Math. J. 22 (1982), 753 - 769.

[6] Nazarov, S. A.: Behavior at infinity of the solutions of Lame and Stokes systems in a sector of a layer (in Russian). Dokl. Akad. Nauk Armenian SSR 87 (1988)4, 156 - 159.

[7] Nazarov, S. A.: Elastic capacity and polarization of a defect in an elastic layer (in Russian). Mekhanika tverd. tela 5 (1990), $57-65$.

[8] Nazarov, S. A.: The spatial structure of the stress field in the neighbourhood of the corner point of a thin plate (in Russian). Prikl. Mat. \& Mech. 55 (1991)4, 523 - 530; Engl. transl. in: J. Appl. Math. \& Mech. 55 (1991)4, 523 - 530.

[9] Nazarov, S. A.: Asymptotics of the solution to a boundary value problem in a thin cylinder with nonsmooth lateral surface (in Russian). Izv. Ross. Akad. Nauk., Ser. Mat. 57 (1993)1, 202 - 239; Engl. transl. in: Math. Izvestiya 42 (1994)1, 183 - 217.

[10] Nazarov, N. A.: Asymptotic expansions at infinity of solutions to theory elasticity problem in a layer (in Russian). Trudy Moskov. Mat. Obschch. 60 (1998), 3 - 97.

[11] Nazarov, S. A. and B. A. Plamenevskii: Asymptotics of the spectrum of the Neumann problem in singularly perturbed thin domains (in Russian). Algebra i Analiz 2 (1990)2, 85 - 111; Engl. transl. in: Leningrad Math. J. 2 (1991)2, 287 - 311.

[12] Nazarov, S. A. and B. A. Plamenevskii: Elliptic Boundary Value Problems in Domains with Piecewise Smooth Boundaries. Berlin: Walter de Gruyter \& Co 1994.

[13] Nazarov, S. A. and K. Pileckas: The Fredholm property of the Neumann problem operator in the domains that are layer-like at infinity (in Russian). Algebra i Analiz8 (1996)6, 57 - 104; Engl. transl. in: St. Petersburg Math. J. 8 (1997)6, 951 - 983.

[14] Nazarov, S. A. and K. Pileckas: On the solvability of the Stokes and Navier-Stokes problems in the domains that are layer-like at infinity. J. Math. Fluid Mech. 1 (1999)1, $78-116$.

[15] Nazarov, S. A. and K. Pileckas: The asymptotic properties of the solutions to the Stokes problem in domains that are layer-like at infinity. J. Math. Fluid. Mech. 1 (1999)2, 131 $-167$.

[16] Nazarov, S. A. and K. Pileckas: Asymptotic conditions at infinity for the Stokes and Navier-Stokes problems in domains with cylindric outlets to infinity. Quaderni di Matematica, Adv. in Fluid Dyn. 4 (1999), 141 - 243.

[17] Nečas, J.: Les mèthodes directes en thèorie des èquations elliptiques. Prague: Academia 1967.

[18] Peetre, J.: Another approach to elliptic boundary problems. Comm. Pure Appl. Math. 14 (1961), $711-731$.

[19] Roitberg, Ya. A. and Z. G. Sheftel: A theorem on homeomorphism for elliptic systems and its application (in Russian). Mat. Sb. 78 (1969), 446 - 472; Engl. transl. in: Math. USSR Sb. 7 (1969), $439-465$.

Received 10.03.2000 